\title{
Level of Micronutrients in Elite Sports: Impact on Muscle Damage, Infections, Sleep Disorders and Fatigue
}

\author{
Klaus Erpenbach ${ }^{1}$, Max C. Erpenbach ${ }^{1}$, Wolfgang Mayer ${ }^{2}$, Uwe Hoffmann ${ }^{3}$, Stefan Mücke ${ }^{1}$
}

\author{
${ }^{1}$ Institut für medizinische Leistungsoptimierung und Trainingssteuerung, Marienstraße 1, 50374 Erftstadt, Germany \\ ${ }^{2}$ Lab4more GmbH Bavariahaus, Augustenstraße 10, 80333 München, Germany \\ ${ }^{3}$ Deutsche Sporthochschule Köln, Am Sportpark Müngersdorf 6, 50933 Köln, Germany
}

Received Date: July 18, 2020; Accepted Date: July 24, 2020; Published Date: August 10, 2020

*Corresponding author: Klaus Erpenbach, Institut für medizinische Leistungsoptimierung und Trainingssteuerung, Marienstraße 1, 50374 Erftstadt, Germany. Email: info@im-lot.org

\section{Abstract}

Summary: Muscle damage, recurrent and serious infections or exercise-induced fatigue especially to the end of the playing period in team sport or to the end of preparation for competition in endurance or single sport are the most common symptoms in elite sport demolishing optimal training results. Are micronutrient deficiencies responsible for these symptoms in elite sport.

Methods: In 111 elite athletes [male: 50 - female: 61 / soccer: 21 - field hockey: 62 - Olympics: 19 - tennis: 5 - motorsports (DTM-Formula1): 4] blood vitamin D, coenzymeQ10, vitamin B1-B2-B6-B12 and folic acid as well as selenium, ferrum and magnesium intraerythrocytary were determined. In all elite athletes the symptoms muscle damage, infections, sleeping disorder and fatigue were correlated. A Spearman-ranking coefficient of correlation, a chi-quadrat-test by Pearson and an independent $\mathrm{t}$-test were used.

Results: In 51, 3\% of all elite athletes $(\mathrm{N}=57 / 111)$ a vitamin Ddeficiency $(<30 \mathrm{ng} / \mathrm{ml})$, in $57 \%(\mathrm{~N}=61 / 107)$ a seleniumdeficiency $(<121 \mu \mathrm{g} / \mathrm{l})$, in $70 \%(\mathrm{~N}=77 / 110)$ a coenzymeQ10deficiency $(<750 \mu \mathrm{g} / \mathrm{l})$ and in $27,1 \%(\mathrm{~N}=19 / 70)$ a magnesium deficiency intraerythrocytary were established. In cases of young player $(<18)$ vitamin $\mathrm{D}$ and of young national player (U16-U21) ferrum intraerythrocytary compared to national A player were poorly supplied [vitamin D: $25,71 \pm 9,58 \mathrm{ng} / \mathrm{ml} \mathrm{vs}$ $35,87 \pm 12,35 \mathrm{ng} / \mathrm{ml}(\mathrm{p}=0,007)-$ ferrum: $407,13 \pm 43,09 \mathrm{mg} / \mathrm{l}$ vs $460,29 \pm 36,69 \mathrm{mg} / \mathrm{l}(\mathrm{p}=0,018)]$. Muscle damages (in $73 \%$ of all cases) significantly occurred in vitamin $\mathrm{D}$ deficiency $[29,13 \pm 9,38 \mathrm{ng} / \mathrm{dl}$ vs $36,27 \pm 12,09 \mathrm{ng} / \mathrm{dl}, \mathrm{p}=0,005]$ and in coenzymeQ10 deficiency $[623,31 \pm 226,31 \mu \mathrm{g} / \mathrm{l}$ vs $732,93 \pm$ $408,19 \mu \mathrm{g} / \mathrm{l}, \mathrm{p}=0,039]$ more frequently. Comparing the settings of vitamin D 40ng/ml $[27,50 \pm 7,85 \mathrm{ng} / \mathrm{ml}$ vs $46,31 \pm 6,71$ $\mathrm{ng} / \mathrm{ml}, \mathrm{OR}=4,53, \mathrm{p}=0,007$ ] muscle damage were observed 4,53 times more frequent in the lower group, whereas the settings of vitaminB1-(thiamine) $<50 \mu \mathrm{g} / \mathrm{l}$ vs $>50 \mu \mathrm{g} / 1[36,45 \pm 7,01 \mu \mathrm{g} / \mathrm{l}$ vs $63,24 \pm 24,33 \mathrm{ng} / \mathrm{ml}, \mathrm{OR}=0,38, \mathrm{p}=0,045]$ showed muscle damage in $62 \%$ less frequent in the lower group. No significances were observed in infection, sleeping disorder or fatigue.

Conclusion: Independantly to the type of sports, except all bvitamines, deficiencies of essential vitamins and trace elements in elite sports were observed. Vitamin D- as well as coenzymeQ10deficiencies and vitaminB1- excess play an important and significant role developing muscle damages. Further studies preventing muscle damages, recurrent infections and fatigue by treating elite athletes with micronutrients to eradicate those deficiencies are necessary.

\section{Introduction}

Micronutrients - vitamins and trace elements - interact with each other. They are essential and are needed daily to optimally maintain all physiological body functions [1-2]. High training effort, tight schedule, unbalanced or incorrect diet, frequent travel and high psychological stress lead to a high consumption of these micronutrients during preparation, which in $12.9 \%$ of cases ends in injuries and in $9.2 \%$ in acute infections and leads to lack of training and cancellation of competitions [3-6]. In major events such as the Olympics, FIFA World Cup or IAAF, 9.6-14\% of participants fail due to injury [5-13]. 5.4-8.9\% of participants suffer from an acute infectious disease, twice as many in winters than in summer [5-13]. The most common acute infections occur in the upper respiratory tract $(46 \%), 25 \%$ in the urogenital system, followed by $21 \%$ with gastrointestinal infections and $8 \%$ skin infections [5-13]. These infections could not be reduced by the "General guidelines for illness prevention in athletes" of the Olympic Committee [14].

For the competitive athlete, micronutrients play a major role in energy production, blood and bone health and antioxidant radical protection [1-2]. The group of B vitamins (thiamine, riboflavin, niacin, pyridoxine, folic acid, cobalamin), coenzyme Q10 and minerals (iron, magnesium, zinc, selenium) are necessary for the mitochondrial oxidation of carbohydrates and fatty acids to energy (ATP) in the respiratory chain and essential for the competitive athletes to maintain their immense energy expenditure [1-2]. For the high turnover of red blood cells (oxygen transport) the athlete needs cellular iron, vitaminB6, -B9 and -B12 [15]. Adequate calcium, magnesium, phosphate and vitamin $\mathrm{D}$ values ensure the maintenance of sufficient bone density and prevent stress fractures [15-17]. Various antioxidants (vitamin A-C-E, selenium, zinc) protect cells and muscle tissue from destruction training and competition-related free oxidative radicals, especially by optimizing the cellular selenium-dependent Glutathione peroxidase and zinc-dependent superoxide dismutase [15]. In taking isolated micronutrients (polyunsaturated fatty acids (PUFA), folic acid, calcium, and antioxidants) seems to reduce muscle injuries and infections [18].

The degree of fatigue a top athlete is highly related to the quality of sleep and stress level in competition [19- 20]. The determination of stress parameters (neurotransmitters: cortisol, serotonin, melatonin) from saliva and urine seem to give first indications of therapeutic impact [20]. Serotonin regulates pain and mood, while melatonin is responsible for sleep induction and is considered a powerful antioxidant for the brain. These neurotransmitters are dependent on their formation on specific 
amino acids (phenylalanine, tyrosine, and tryptophan), B vitamins (especially pyridoxine) and magnesium [21]. Epidemiological, prevention or therapy studies are not known yet.

The aim of this study is to evaluate the micronutrient status of top athletes of various sports and to evaluate possible interrelationship with fatigue, sleep disorders, susceptibility to infections and muscle injuries. Are there clear differences between deficiencies of these vitamins and trace elements and the athletes' complaints measurable?.

\section{Methods}

\section{Attendee}

111 top athletes from various sports (team sports: field hockey and soccer - individual sports: athletics, tennis, motorsports) were included in the study to optimize their performance. All participants gave their agreement and were evaluated according to a questionnaire (sleep disorders, fatigue, muscle injuries, susceptibility to infections). Fatigue was defined as morning fatigue and lack of drive despite 7-hour sleep. Muscle injuries included all medically confirmed muscle fiber tears such a muscle bundle tears and tendon muscle tears. Susceptibility to infection was defined as at least 2 medically confirmed viral and/or bacterial infections or more per annum. Sleep disorders included both difficulty falling-asleep $(>15 \mathrm{~min}$ duration to sleep) and difficulty sleeping-through. In all athletes, vitamin B1 -B2 -B6 -B12, folic acid, vitamin D, intracellular iron-selenium-magnesium, and coenzyme Q10 were examined in the blood and correlated with the symptoms.

\section{Vitamins B1 (Thiamine), B2 (Riboflavin), B6 (Pyridoxin)} (EDTA blood) and coenzyme Q10 (Serum)

The determination of the $\mathrm{B}$ vitamins $\mathrm{B} 1$ (thiamine), B2 (riboflavin), B6 (pyridoxin) from EDTA blood and Q10 in serum was performed by high performance liquid chromatography (HPLC) with commercial kits (Chromsystems, Gräfelfing, Germany) according to manufacturer's specifications (B1 and B6 - Order No. 51052 / B2 - Order No. 37000 / Q10 - Order No. 68000).

\section{Vitamin B9 (Folic acid) and B12 (Cobalamin) (Serum)}

The determination of vitamins B9 (folic acid) and B12 (cobalamin) was performed according to the manufacturer's specifications as a competitive immunoassay with an automated two-step procedure on the Labor mat Immulite-2000XPI of Siemens Healthcare, Erlangen, Germany (B9 - Order No. 6605868 / B12 - Order No. 6608026).

\section{Vitamin D 25-OH (Serum)}

The quantitative determination of the absolute concentration of $25-\mathrm{OH}$ vitamin $\mathrm{D}$ in the serum was performed according to the manufacturer's specifications by using a direct competitive chemiluminescence immunoassay (CLIA) at the laboratory machine Liasion-XL of Diasorin, Dietzenbach, Germany (Order No. 310600).

\section{Magnesium and Selenium (EDTA blood)}

The determination of magnesium and selenium in whole blood was performed by atomic absorption spectrometry (AAS).

\section{Iron (EDTA blood)}

The iron content in whole blood was determined by mass spectrometry with inductively coupled plasma (ICPMS).

\section{Statistical Analysis}

The data were evaluated statistically with the IBM®SPSS $®$ software 25. Spearman Rho was used to calculate the correlation between the different parameters. A Pearson chi-square $\chi^{2} 4$ field test was used to compare the frequency of symptoms and age groups, and the Odds Ratio (OR) was calculated. The $95 \%$ confidence interval (CI) was calculated according to

$$
K I 95 \%=\exp \left[\ln (\mathrm{OR}) \pm 1.96 \sqrt{ } \sum 1 \mathrm{fi} \ln (\mathrm{OR})\right]
$$

For 2-group mean value comparisons, a level test was performed to examine variance homogeneity followed by an independent $\mathrm{T}$-test for homogeneous $(\mathrm{P}>0.1)$ or homogeneous variance with bilateral questions. For $k>2$ groups or for combined influences of the different patient groups and the respective symptoms, a one- or two factor variance analysis (ANOVA) was applied. Post-hoc mean values were equalized with the two-sided Sidak test. For $p \leq 0.05$ the results were described as significant, for $\mathrm{p} \leq 0.01$ as highly significant.

Results

(Table 1) illustrates the characteristics of the study population. $73 \%$ of all top athletes complained about muscle injuries, $44 \%$ fatigue, $40 \%$ infections and $32 \%$ sleep disorders. Female athletes high significantly were worse supplied within trace cellular selenium $(109 \pm 26.01$ vs. $125.4 \pm 30.51 \mu \mathrm{g} / \mathrm{l}$, $\mathrm{p}<0.0005)$, intracellular magnesium $(1.3 \pm 0.11$ vs. $1.4 \pm 0.13$ $\mathrm{mmol} / \mathrm{l}, \mathrm{p}<0.024)$ and intracellular iron (403.6 \pm 35.72 vs. 477 $\pm 31.1 \mathrm{mg} / \mathrm{l}, \mathrm{p}<0.0005)$ respectively than their male counterparts. In total population, the average value of selenium was $116.95 \pm 29.08 \mu \mathrm{g} / \mathrm{l}$ (norm: $121-168 \mu \mathrm{g} / \mathrm{l}$ ) and Q10 was 653.21 $\pm 289.58 \mu \mathrm{g} / \mathrm{l}$ (norm: $750-1200 \mu \mathrm{g} / \mathrm{l}$ ) below the norm. Young national players U16-U18 significantly suffered 3-times more from muscle injuries than the A national players [86\% (18/21) vs. $67 \%(18 / 27)$ - OR $=3.00 ; 95 \%$ CI: $1.50-4.50, p=0.022]$. No differences were found in infections, fatigue and sleep disorders. The vitamin D value of $51.3 \%$ (57/111) of the athletes determined $<30 \mathrm{ng} / \mathrm{ml}$, with $13.5 \%$ (15/111) having a vitamin D deficiency $(<20 \mathrm{ng} / \mathrm{ml})$ and $37.8 \%$ (42/111) having an inadequate vitamin D supply (> 20-30 ng/ml).

(Table 2) shows the comparison of youth vs. adults. In contrast to adults, adolescents are more badly supplied with almost all micronutrients (except vitamin B9 = folic acid). For vitamin $\mathrm{D}(25.71 \pm 9.58 \mathrm{ng} / \mathrm{ml}$ vs. $35.78 \pm 12.35 \mathrm{ng} / \mathrm{ml}-$ $\mathrm{p}=0.007$ ) (Figure 1) and intercellular iron $(407.13 \pm 43.09 \mathrm{mg} / \mathrm{l}$ vs. $460.29 \pm 36.69 \mathrm{mg} / \mathrm{l}-\mathrm{p}=0.018)$ the youth players respectively the young national players U16-U18 are significantly worse supplied compared to the A-national players. The percentage of youth players within sufficient or inadequate vitamin D supply was $64.4 \%$ (29/45), with $22.2 \%$ $(10 / 45)$ youth players showing vitamin D deficiency $(<20$ $\mathrm{ng} / \mathrm{ml}$ ) and $42.4 \%(19 / 45)$ showing in adequate vitamin D supply $(20-30 \mathrm{ng} / \mathrm{ml})$. As a result, youth players have a significantly 2.46-fold higher risk of developing vitamin D deficiency $(29 / 45$ vs $28 / 66$, OR $=2.46,95 \%$ CI: $1.67-3.24$, $\mathrm{p}<0.023)$ and a 2.72 -fold higher risk of developing intracellular iron deficiency $(14 / 30$ vs 9/37, OR $=2.72,95 \%$ CI: $1.68-3.76$, $\mathrm{p}<0.055)$ than adults.

In (Table 3) the values of the athletes in a group comparison are divided into groups below and above international recommendations [15, 22-27] in correlation to the symptoms. Deficiencies of micronutrients showed no effect regarding to sleep disorders. The probability to fatigue only tends to rise by selenium deficiency $(\mathrm{p}=0,091)$. Q10-deficiency as well tends to raise the probability of infections $(\mathrm{p}=0,065)$. Muscle damage significantly develop to vitamin-d-deficiency $(\mathrm{p}=0,007)$ and vitamin-B1-excess $(\mathrm{p}=0,045)$, respectively, and tends to rise by vitamin-B6-excess $(\mathrm{p}=0,058)$. 


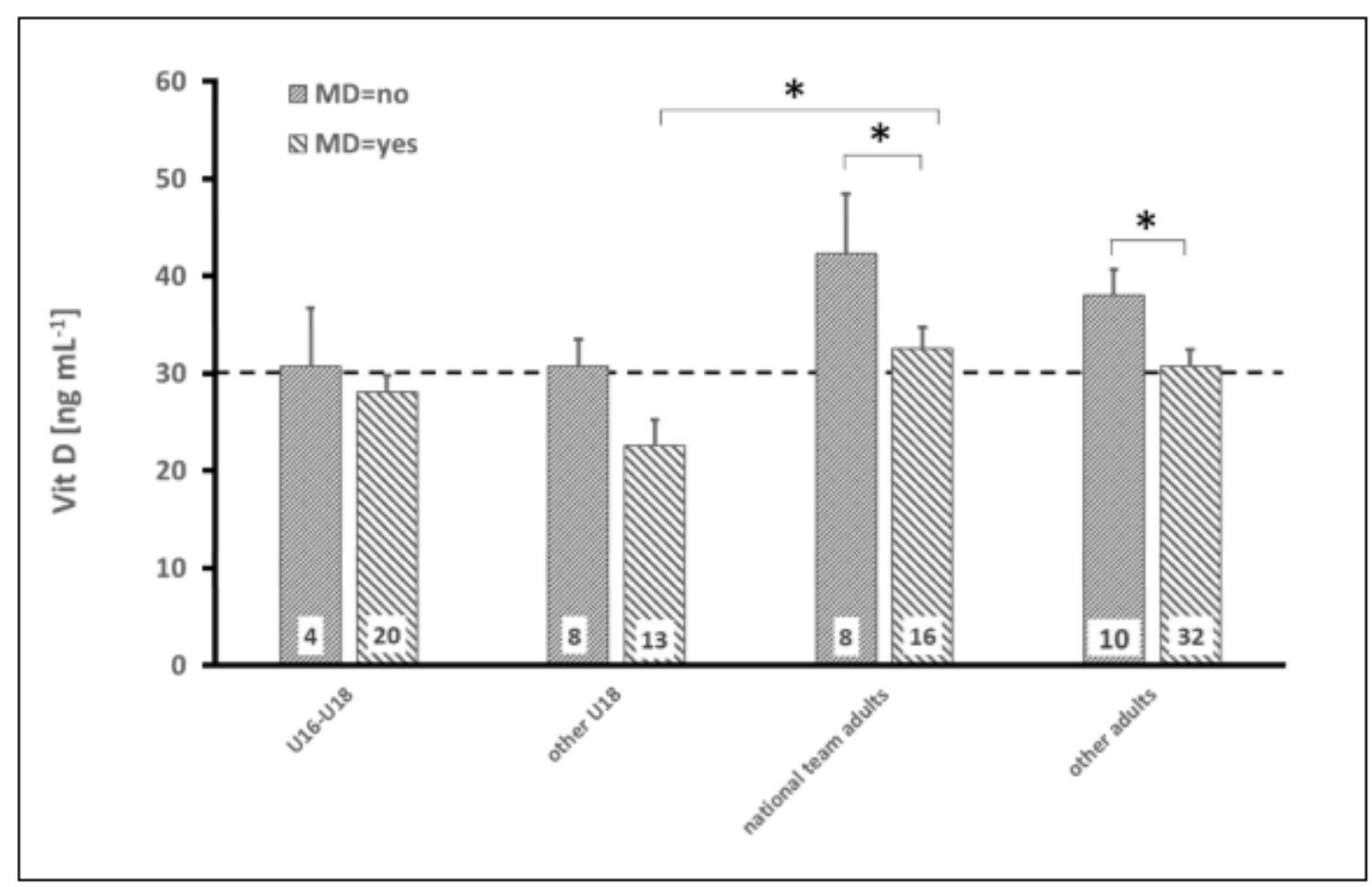

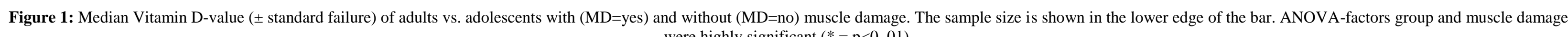
were highly significant $(*=p<0,01)$.

\begin{tabular}{|c|c|c|c|c|c|c|c|c|c|c|c|c|c|c|c|}
\hline & Age & Infect & $\begin{array}{l}\text { Muscle } \\
\text { Injury }\end{array}$ & Fatigue & $\begin{array}{c}\text { Sleep } \\
\text { Disorder }\end{array}$ & $\begin{array}{c}\text { VitD } \\
30-80 \\
\text { ng/ml } \\
\end{array}$ & $\begin{array}{c}\text { Selenium } \\
121-168 \\
\mu \mathrm{g} / \mathrm{l}\end{array}$ & $\begin{array}{c}\text { Magnesium } \\
1,29-1,69 \\
\mathrm{mmol} / \mathrm{l}\end{array}$ & $\begin{array}{c}\text { Iron } \\
420-460 \\
\text { mg/l } \\
\end{array}$ & $\begin{array}{c}\text { Qu10 } \\
750-1200 \\
\mu \mathrm{g} / \mathrm{l}\end{array}$ & $\begin{array}{c}\text { VitB1 } \\
30-90 \\
\mu \mathrm{g} / \mathrm{l}\end{array}$ & $\begin{array}{c}\text { VitB2 } \\
137-270 \\
\mu \mathrm{g} / \mathrm{l}\end{array}$ & $\begin{array}{c}\text { VitB6 } \\
5-30 \\
\mu \mathrm{g} / \mathrm{l} \\
\end{array}$ & $\begin{array}{c}\text { VitB9 } \\
4,6-18,7 \\
\mathrm{ng} / \mathrm{ml} \\
\end{array}$ & $\begin{array}{c}\text { VitB12 } \\
200-950 \\
\text { pg/ml }\end{array}$ \\
\hline \multirow{3}{*}{ Total $(n=111)$} & 22,34 & $44 / 111$ & $81 / 111$ & $49 / 111$ & $36 / 111$ & 31,1 & $\begin{array}{c}121-168 \\
\mu \mathrm{g} / \mathrm{l}\end{array}$ & 1,4 & 438,7 & 653,2 & 54,8 & 170,7 & 25,1 & 8,9 & 465,6 \\
\hline & $\pm 7,43$ & $40 \%$ & $73 \%$ & $44 \%$ & 32 & $\pm 10,61$ & $\pm 29,08$ & $\pm 0,15$ & $\pm 49,77$ & $\pm 289,58$ & $\pm 21,62$ & $\pm 36,06$ & $\pm 15,24$ & $\pm 4,35$ & $\pm 219,57$ \\
\hline & & & & & & $(\mathrm{n}=111)$ & $(\mathrm{n}=107)$ & $(\mathrm{n}=70)$ & $(n=67)$ & $(\mathrm{n}=111)$ & $(\mathrm{n}=90)$ & $(\mathrm{n}=90)$ & $(\mathrm{n}=88)$ & $(\mathrm{n}=100)$ & $(n=92)$ \\
\hline \multirow{3}{*}{ Female $(n=61)$} & 20,13 & $24 / 61$ & $44 / 61$ & $30 / 61$ & $17 / 61$ & 31,9 & 109,8 & 1,3 & 403,6 & 642,7 & 57,0 & 168,2 & 23,0 & 9,1 & 427,1 \\
\hline & $\pm 6,16$ & $39 \%$ & $72 \%$ & $49 \%$ & $28 \%$ & $\pm 9,92$ & $\pm 26,01$ & $\pm 0,11$ & $\pm 35,72$ & $\pm 241,12$ & $\pm 26,29$ & $\pm 37,34$ & $\pm 18,91$ & $\pm 4,63$ & $\pm 194,80$ \\
\hline & & & & & & $(\mathrm{n}=61)$ & $(\mathrm{n}=58)$ & $(\mathrm{n}=35)$ & $(\mathrm{n}=35)$ & $(\mathrm{n}=60)$ & $(\mathrm{n}=46)$ & $(\mathrm{n}=47)$ & $(\mathrm{n}=45)$ & $(\mathrm{n}=50)$ & $(\mathrm{n}=48)$ \\
\hline \multirow{3}{*}{ Male $(n=50)$} & 25,04 & $20 / 50$ & $37 / 50$ & $19 / 50$ & $19 / 50$ & 30,0 & 125,4 & 1,4 & 477,0 & 665,8 & 52,6 & 173,5 & 27,3 & 8,7 & 506,4 \\
\hline & \pm 8 & $40 \%$ & $74 \%$ & $38 \%$ & $38 \%$ & $\pm 11,42$ & $\pm 30,51$ & $\pm 0,13$ & $\pm 31,10$ & $\pm 340,93$ & $\pm 15,29$ & $\pm 34,82$ & $\pm 17,69$ & $\pm 4,08$ & $\pm 239,15$ \\
\hline & & & & & & $(\mathrm{n}=50)$ & $(\mathrm{n}=49)$ & $(\mathrm{n}=35)$ & $(\mathrm{n}=32)$ & $(\mathrm{n}=50)$ & $(\mathrm{n}=44)$ & $(\mathrm{n}=43)$ & $(\mathrm{n}=43)$ & $(\mathrm{n}=50)$ & $(\mathrm{n}=44)$ \\
\hline Difference $\mathbf{m} / \mathbf{f}$ & & n.s. & n.s. & n.s. & n.s. & n.s. & $\mathrm{p}<0,005$ & $\mathrm{p}<0,024$ & $\mathrm{p}<0,0005$ & n.s. & n.s. & n.s. & n.s. & n.s. & n.s. \\
\hline \multirow{3}{*}{ Soccer $(n=21)$} & 22,9 & $5 / 21$ & $17 / 21$ & $4 / 21$ & $3 / 21$ & 32,2 & 134,2 & 1,4 & 471,9 & 703,6 & 48,6 & 180,0 & 29,3 & 8,3 & 592,2 \\
\hline & $\pm 4,05$ & $24 \%$ & $81 \%$ & $19 \%$ & $14 \%$ & $\pm 13,29$ & $\pm 26,2$ & $\pm 0,15$ & $\pm 31,86$ & $\pm 468,48$ & $\pm 18,01$ & $\pm 31,32$ & $\pm 16,6$ & $\pm 4,91$ & $\pm 231,97$ \\
\hline & & & & & & $(\mathrm{n}=21)$ & $(\mathrm{n}=20)$ & $(n=19)$ & $(n=17)$ & $(n=20)$ & $(\mathrm{n}=16)$ & $(\mathrm{n}=16)$ & $(\mathrm{n}=15)$ & $(n=18)$ & $(\mathrm{n}=17)$ \\
\hline \multirow{3}{*}{ Field Hockey $(\mathrm{n}=62)$} & 19,37 & $22 / 62$ & $45 / 62$ & $26 / 62$ & $17 / 62$ & 31,6 & 104,2 & 1,4 & 409,6 & 623,6 & 55,8 & 168,3 & 22,9 & 8,0 & 379,6 \\
\hline & $\pm 4,62$ & $36 \%$ & $73 \%$ & $42 \%$ & $27 \%$ & $\pm 9,99$ & $\pm 23,49$ & $\pm 0,11$ & $\pm 33,67$ & $\pm 217,25$ & $\pm 11,77$ & $\pm 29,85$ & $\pm 17,41$ & $\pm 2,95$ & $\pm 135,22$ \\
\hline & & & & & & $(\mathrm{n}=62)$ & $(n=60)$ & $(n=27)$ & $(\mathrm{n}=29)$ & $(\mathrm{n}=62)$ & $(\mathrm{n}=54)$ & $(\mathrm{n}=53)$ & $(\mathrm{n}=53)$ & $(\mathrm{n}=55)$ & $(\mathrm{n}=54)$ \\
\hline \multirow{2}{*}{$\begin{array}{c}\text { Olympia Participants } \\
(n=19)\end{array}$} & $\pm 10,86$ & $58 \%$ & $68 \%$ & $74 \%$ & $53 \%$ & $\pm 10,58$ & $\pm 32,12$ & $\pm 0,11$ & $\pm 68,55$ & $\pm 291,55$ & $\pm 14,03$ & $\pm 52,45$ & $\pm 22,77$ & $\pm 5,65$ & $\pm 273,09$ \\
\hline & & & & & & $(\mathrm{n}=19)$ & $(\mathrm{n}=18)$ & $(\mathrm{n}=15)$ & $(n=14)$ & $(n=19)$ & $(\mathrm{n}=12)$ & $(\mathrm{n}=13)$ & $(\mathrm{n}=12)$ & $(\mathrm{n}=18)$ & $(\mathrm{n}=13)$ \\
\hline \multirow[t]{3}{*}{ Tennis $(\mathbf{n}=5)$} & 21,6 & $3 / 5$ & $4 / 5$ & $2 / 5$ & $2 / 5$ & 27,1 & 133,8 & 1,3 & 430,5 & 638,8 & 48,5 & 217,5 & 76,3 & 13,0 & 589,8 \\
\hline & $\pm 6,5$ & $60 \%$ & $80 \%$ & $40 \%$ & $40 \%$ & $\pm 10,84$ & $\pm 26,93$ & $\pm 0,06$ & $\pm 27,6$ & $\pm 254,55$ & $\pm 14,27$ & $\pm 12,71$ & $\pm 71,77$ & $\pm 5,96$ & $\pm 180,23$ \\
\hline & & & & & & $(\mathrm{n}=5)$ & $(\mathrm{n}=5)$ & $(\mathrm{n}=5)$ & $(\mathrm{n}=4)$ & $(\mathrm{n}=5)$ & $(\mathrm{n}=4)$ & $(\mathrm{n}=4)$ & $(\mathrm{n}=4)$ & $(\mathrm{n}=5)$ & $(\mathrm{n}=4)$ \\
\hline \multirow[t]{3}{*}{ Motor $(n=4)$} & 34,75 & $3 / 4$ & $2 / 4$ & $3 / 4$ & $4 / 4$ & 29,0 & 128,3 & 1,5 & 481,7 & 552,5 & 46,0 & 187,5 & 16,5 & 10,0 & 560,0 \\
\hline & $\pm 6,85$ & $75 \%$ & $50 \%$ & $75 \%$ & $100 \%$ & $\pm 6,05$ & $\pm 20,6$ & $\pm 0,11$ & $\pm 17,93$ & $\pm 148,08$ & $\pm 15,1$ & $\pm 34,77$ & $\pm 3,6$ & $\pm 6,5$ & $\pm 206,11$ \\
\hline & & & & & & $(\mathrm{n}=4)$ & $(n=4)$ & $(n=4)$ & $(\mathrm{n}=3)$ & $(\mathrm{n}=4)$ & $(\mathrm{n}=4)$ & $(\mathrm{n}=4)$ & $(\mathrm{n}=4)$ & $(\mathrm{n}=4)$ & $(\mathrm{n}=4)$ \\
\hline \multirow{3}{*}{ National $(n=27)$} & 23,59 & $8 / 27$ & $18 / 27$ & $11 / 27$ & $8 / 27$ & 34,4 & 121,5 & 1,4 & 457,5 & 738,3 & 53,2 & 172,4 & 30,8 & 8,7 & 447,7 \\
\hline & $\pm 5,35$ & $30 \%$ & $67 \%$ & $41 \%$ & $30 \%$ & $\pm 12,94$ & $\pm 31,61$ & $\pm 0,15$ & $\pm 37,13$ & $\pm 420,2$ & $\pm 8,85$ & $\pm 32,8$ & $\pm 25,19$ & $\pm 5,61$ & $\pm 199,61$ \\
\hline & & & & & & $(\mathrm{n}=27)$ & $(\mathrm{n}=25)$ & $(\mathrm{n}=15)$ & $(\mathrm{n}=16)$ & $(\mathrm{n}=27)$ & $(\mathrm{n}=23)$ & $(\mathrm{n}=22)$ & $(\mathrm{n}=22)$ & $(\mathrm{n}=27)$ & $(\mathrm{n}=23)$ \\
\hline \multirow[t]{3}{*}{ U16 $(n=6)$} & 13,67 & $1 / 6$ & $5 / 6$ & $1 / 6$ & $0 / 6$ & 29,9 & 100,1 & 1,4 & 367,8 & 496,7 & 55,3 & 160,3 & 15,5 & 8,6 & 388,3 \\
\hline & $\pm 0,82$ & $17 \%$ & $83 \%$ & $17 \%$ & 0 & $\pm 8,04$ & $\pm 36,25$ & $\pm 0,07$ & $\pm 5,32$ & $\pm 187,26$ & $\pm 10,89$ & $\pm 5,86$ & $\pm 4,43$ & $\pm 3,28$ & $\pm 99,3$ \\
\hline & & & & & & $(n=6)$ & $(\mathrm{n}=6)$ & $(\mathrm{n}=4)$ & $(\mathrm{n}=6)$ & $(\mathrm{n}=6)$ & $(n=3)$ & $(n=3)$ & $(n=3)$ & $(n=3)$ & $(n=3)$ \\
\hline \multirow[t]{3}{*}{ U18 (n=15) } & 16,47 & $4 / 15$ & $13 / 15$ & $4 / 15$ & $2 / 15$ & 28,9 & 108,5 & 1,4 & 417,8 & 602,1 & 55,1 & 168,8 & 21,7 & 9,0 & 412,5 \\
\hline & $\pm 0,64$ & $27 \%$ & $87 \%$ & $27 \%$ & $13 \%$ & $\pm 8,32$ & $\pm 30,12$ & $\pm 0,12$ & $\pm 42,53$ & $\pm 241,6$ & $\pm 13,07$ & $\pm 47,2$ & $\pm 7,17$ & $\pm 3,6$ & $\pm 170,18$ \\
\hline & & & & & & $(n=15)$ & $(\mathrm{n}=15)$ & $(\mathrm{n}=10)$ & $(\mathrm{n}=9)$ & $(n=15)$ & $(\mathrm{n}=12)$ & $(n=12)$ & $(\mathrm{n}=11)$ & $(n=12)$ & $(n=12)$ \\
\hline
\end{tabular}

Table 1: Micronutrients and symptoms: infection, muscle damage, fatigue, sleep disorder correlated to gender and sports.

\begin{tabular}{|c|c|c|c|c|c|c|c|c|c|c|}
\hline & $\begin{array}{l}\text { VitD } \\
30-80 \mathrm{ng} / \mathrm{ml}\end{array}$ & $\begin{array}{l}\text { Selenium } \\
\text { 121-168 } \mu \mathrm{g} / \mathrm{l}\end{array}$ & $\begin{array}{c}\text { Magnesium } \\
1,29-1,69 \\
\mathrm{mmol} / \mathrm{l}\end{array}$ & $\begin{array}{c}\text { Iron } \\
420-460 \\
\mathrm{mg} / \mathrm{l}\end{array}$ & $\begin{array}{c}\text { Qu10 } \\
750-1200 \\
\mu \mathrm{g} / \mathrm{l}\end{array}$ & $\begin{array}{c}\text { VitB1 } \\
30-90 \\
\mu \mathrm{g} / \mathrm{l}\end{array}$ & $\begin{array}{c}\text { VitB2 } \\
137-270 \mu \mathrm{g} / \mathrm{l}\end{array}$ & $\begin{array}{l}\text { VitB6 } \\
5-30 \\
\mu \mathrm{g} / \mathrm{l}\end{array}$ & $\begin{array}{c}\text { VitB9 } \\
4,6-18,7 \\
\mathrm{ng} / \mathrm{ml}\end{array}$ & $\begin{array}{c}\text { VitB12 } \\
200-950 \\
\text { pg/ml }\end{array}$ \\
\hline Significance & $p=0,006$ & & & $p=0,008$ & & & & & & \\
\hline Total & 31,06 & 116,95 & 1,37 & 438,67 & 653,21 & 54,82 & 170,68 & 25,13 & 8,87 & 465,57 \\
\hline \multirow[t]{2}{*}{$(n=111)$} & $\pm 10,61$ & $\pm 29,08$ & $\pm 0,15$ & $\pm 49,77$ & $\pm 289,58$ & $\pm 21,62$ & $\pm 36,06$ & $\pm 15,24$ & $\pm 4,35$ & $\pm 219,57$ \\
\hline & $(\mathrm{n}=111)$ & $(\mathrm{n}=107)$ & $(n=70)$ & $(\mathrm{n}=67)$ & $(\mathrm{n}=111)$ & $(\mathrm{n}=90)$ & $(\mathrm{n}=90)$ & $(\mathrm{n}=88)$ & $(n=100)$ & $(\mathrm{n}=92)$ \\
\hline \multicolumn{2}{|c|}{ National Player 35, $78^{*}$} & 123,23 & 1,42 & $460,29 *$ & 751,71 & 53,14 & 177,11 & 32,21 & 8,79 & 459,55 \\
\hline Adults & $\pm 12,35$ & $\pm 32,9$ & $\pm 0,15$ & $\pm 36,69$ & $\pm 441,80$ & $\pm 9,52$ & $\pm 29,20$ & $\pm 26,90$ & $\pm 5,92$ & $\pm 211,78$ \\
\hline$(n=24)$ & $(\mathrm{n}=24)$ & $(\mathrm{n}=22)$ & $(n=13)$ & $(n=14)$ & $(n=24)$ & $(\mathrm{n}=20)$ & $(\mathrm{n}=19)$ & $(n=19)$ & $(n=24)$ & $(\mathrm{n}=20)$ \\
\hline
\end{tabular}




\begin{tabular}{|c|c|c|c|c|c|c|c|c|c|c|}
\hline \multirow{2}{*}{\multicolumn{2}{|c|}{ National Player 28, 53}} & & & & & & & & & \\
\hline & & $106,4 /$ & 1,39 & $40 /, 13{ }^{\mathrm{N}}$ & $5 / 9,25$ & 54,84 & 163,03 & 20,00 & 8,81 & 401,17 \\
\hline U16 - U18 & $\pm 8,2$ & $\pm 29,71$ & $\pm 0,11$ & $\pm 43,09$ & $\pm 233,63$ & $\pm 11,18$ & $\pm 42,29$ & $\pm 6,52$ & $\pm 3,23$ & $\pm 142,73$ \\
\hline$(n=24)$ & $(n=24)$ & $(n=24)$ & $(n=16)$ & $(n=15)$ & $(n=24)$ & $(n=18)$ & $(n=18)$ & $(n=17)$ & $(n=18)$ & $(\mathrm{n}=18)$ \\
\hline \multirow[t]{2}{*}{ Significance } & & & & $* p=0,018$ & & & & & & \\
\hline & & & & $\# p=0,026$ & & & & & & \\
\hline other U18 & $25,71^{*}$ & 111,76 & 1,31 & 427,5 & 592,05 & 49,93 & 156 & 23,76 & 10,8 & 487,18 \\
\hline \multirow[t]{2}{*}{$(n=21)$} & $\pm 9,58$ & $\pm 21,31$ & $\pm 0,12$ & $\pm 50,58$ & $\pm 275,72$ & $\pm 17,69$ & $\pm 43,90$ & $\pm 18,95$ & $\pm 4,51$ & $\pm 265,42$ \\
\hline & $(\mathrm{n}=21)$ & $(\mathrm{n}=20)$ & $(n=14)$ & $(n=14)$ & $(\mathrm{n}=21)$ & $(\mathrm{n}=16)$ & $(\mathrm{n}=17)$ & $(n=16)$ & $(\mathrm{n}=18)$ & $(\mathrm{n}=17)$ \\
\hline Significance & $* p=0,007$ & & & & & & & & & \\
\hline Others & 32,47 & 122,24 & 1,37 & $452,29 \#$ & 668,31 & 49,93 & 178,06 & 24,11 & 8,07 & 488,84 \\
\hline Adults & $\pm 9,67$ & $\pm 28,62$ & $\pm 0,13$ & $\pm 50,67$ & $\pm 289,58$ & $\pm 21,62$ & $\pm 30,07$ & $\pm 15,88$ & $\pm 3,39$ & $\pm 233,22$ \\
\hline$(n=42)$ & $(n=42)$ & $(\mathrm{n}=41)$ & $(\mathrm{n}=27)$ & $(n=24)$ & $(\mathrm{n}=42)$ & $(\mathrm{n}=35)$ & $(n=36)$ & $(n=36)$ & $(n=40)$ & $(n=37)$ \\
\hline
\end{tabular}

Table 2: Value of micronutrients comparing adults and youth.

\begin{tabular}{|c|c|c|c|c|c|c|c|c|c|c|c|c|c|c|c|c|c|c|c|c|}
\hline \multirow[b]{2}{*}{ A } & \multicolumn{2}{|c|}{$\begin{array}{c}\text { VitD } \\
30-80 \mathrm{ng} / \mathrm{ml}\end{array}$} & \multicolumn{2}{|c|}{$\begin{array}{c}\text { Selenium } \\
121-168 \mu \mathrm{g} / \mathrm{l}\end{array}$} & \multicolumn{2}{|c|}{$\begin{array}{c}\text { Magnesium } \\
\text { 1, 29-1, } 69 \mathrm{mmol} / \mathrm{l}\end{array}$} & \multicolumn{2}{|c|}{$\begin{array}{c}\text { Iron 420-460 } \\
\mathrm{mg} / \mathrm{l}\end{array}$} & \multicolumn{2}{|c|}{$\begin{array}{c}\text { Qu10 750-1200 } \\
\mu \mathrm{g} / \mathrm{l}\end{array}$} & \multicolumn{2}{|c|}{$\begin{array}{c}\text { VitB1 30-90 } \\
\mu \mathrm{g} / \mathrm{l}\end{array}$} & \multicolumn{2}{|c|}{$\begin{array}{c}\text { VitB2 } \\
137-270 \mu \mathrm{g} / \mathrm{l}\end{array}$} & \multicolumn{2}{|c|}{$\begin{array}{c}\text { VitB6 5-30 } \\
\mu \mathrm{g} / \mathrm{l}\end{array}$} & \multicolumn{2}{|c|}{$\begin{array}{c}\text { VitB9 4, 6-18, } 7 \\
\text { ng/ml }\end{array}$} & \multicolumn{2}{|c|}{$\begin{array}{c}\text { VitB12 200-950 } \\
\mathrm{pg} / \mathrm{ml}\end{array}$} \\
\hline & $\leq 40$ & & $\leq 140$ & & $\leq 1,40$ & & $\leq 420$ & & $\leq 1000$ & & $\leq 50$ & & $\leq 200$ & & $\leq 20$ & & $\leq 15$ & & $\leq 400$ & \\
\hline B & $>40$ & $\mathrm{n}$ & $>140$ & $\mathrm{n}$ & $>1,40$ & $\mathrm{n}$ & $>420$ & $\mathrm{n}$ & $>1000$ & $\mathrm{n}$ & $>50$ & $\mathrm{n}$ & $>200$ & $\mathrm{n}$ & $>20$ & $\mathrm{n}$ & $>15$ & $\mathrm{n}$ & $>400$ & $\mathrm{n}$ \\
\hline Total & 27,96 & 95 & 111,2 & 97 & 1,29 & 43 & 385 & 22 & 600 & $2^{10}$ & 36,17 & 23 & 157,1 & 70 & 13.53 & 44 & 8,05 & 94 & 331 & 59 \\
\hline \pm SD & $\pm 7,53$ & & $\pm 23,40$ & & $\pm 0,08$ & & $\pm 37,74$ & & $\pm 187,33$ & & $\pm 6,30$ & & $\pm 28,12$ & & $\pm 3,78$ & & $\pm 2,8$ & & $\pm 86,95$ & \\
\hline Total & 49,43 & 16 & 172,5 & 10 & 1,50 & 27 & 465 & 45 & 1328 & 8 & 61,23 & 67 & 218,4 & 20 & 36,72 & 44 & 21,63 & 6 & 704 & 33 \\
\hline \multirow[t]{2}{*}{ \pm SD } & $\pm 7,04$ & & $\pm 22,61$ & & $\pm 0,82$ & & $\pm 32,58$ & & $\pm 488,27$ & & $\pm 21,31$ & & $\pm 11,66$ & & $\pm 19,78$ & & $\pm 4,52$ & & $\pm 177,23$ & \\
\hline & \multicolumn{20}{|c|}{ Sleep Disorder } \\
\hline Yes (A) & 28,74 & 32 & 111,1 & 29 & 1,31 & 12 & 373 & 5 & 624 & 33 & 37,05 & 10 & 161,9 & 25 & 13,24 & 14 & 7,84 & 31 & 333 & 20 \\
\hline \pm SD & $\pm 5,74$ & & $\pm 20,62$ & & $\pm 0,05$ & & $\pm 57,28$ & & $\pm 168,49$ & & $\pm 4,34$ & & $\pm 27,55$ & & $\pm 3,95$ & & $\pm 2,90$ & & $\pm 91,07$ & \\
\hline Yes (B) & 50,43 & 4 & 173,0 & 7 & 1,52 & 24 & 463 & 31 & 1153 & 3 & 66,88 & 26 & 219,0 & 11 & 35,33 & 22 & 20,53 & 5 & 665 & 16 \\
\hline$\pm \mathrm{SD}$ & $\pm 8,08$ & & $\pm 15,48$ & & $\pm 0,09$ & & $\pm 32,61$ & & $\pm 94,69$ & & $\pm 33,96$ & & $\pm 12,33$ & & $\pm 15,82$ & & $\pm 5,28$ & & $\pm 165,12$ & \\
\hline & $O D=1,52-$ & & $\begin{array}{c}O D=0 \\
43-\end{array}$ & & $O D=0,71-$ & & $O D=0,55$ & & $O D=1,00-$ & & $O D=1,83$ & & $\begin{array}{c}O D=1 \\
52-\end{array}$ & & $O D=0,95$ & & $\begin{array}{c}O D=1 \\
18\end{array}$ & & $\begin{array}{c}O D=1 \\
15\end{array}$ & \\
\hline & $p=0,354$ & & $p=0,117$ & & $p=0,275$ & & $p=0,205$ & & $p=0,607$ & & $p=0,154$ & & $p=0,226$ & & $p=0,540$ & & $p=0,506$ & & $p=0,442$ & \\
\hline No (A) & 27,57 & 63 & 111,3 & 68 & 1,28 & 31 & 388 & 17 & 589 & 66 & 35,49 & 13 & 154,4 & 45 & 13,67 & 30 & 8,15 & 63 & 330 & 39 \\
\hline \pm SD & $\pm 8,31$ & & $\pm 24,15$ & & $\pm 0,09$ & & $\pm 21,32$ & & $\pm 115,86$ & & $\pm 7,59$ & & $\pm 28,39$ & & $\pm 3,76$ & & $\pm 2,77$ & & $\pm 85,96$ & \\
\hline No (B) & 49,30 & 12 & 171,8 & 7 & 1,49 & 44 & 467 & 58 & 1433 & 6 & 58,64 & 62 & 218,1 & 30 & 37,60 & 45 & 23,85 & 12 & 724 & 36 \\
\hline$\pm \mathrm{SD}$ & $\pm 7,03$ & & $\pm 33,66$ & & $\pm 0,08$ & & $\pm 33,06$ & & $\pm 613,21$ & & $\pm 11,48$ & & $\pm 11,82$ & & $\pm 22,16$ & & $\pm 1,91$ & & $\pm 183,54$ & \\
\hline & & & & & & & & & & & tigue & & & & & & & & & \\
\hline Yes (A) & 28,35 & 42 & 116,1 & 40 & 1,29 & 20 & 382 & 11 & 635 & 45 & 35,95 & 10 & 154,4 & 29 & 12,48 & 16 & 7,75 & 41 & 312 & 25 \\
\hline \pm SD & $\pm 6,60$ & & $\pm 18,17$ & & $\pm 0,08$ & & $\pm 39,88$ & & $\pm 193,34$ & & $\pm 4,73$ & & $\pm 29,36$ & & $\pm 3,66$ & & $\pm 3,00$ & & $\pm 89,57$ & \\
\hline Yes (B) & 50,53 & 7 & 169,0 & 9 & 1,51 & 29 & 457 & 38 & 1123 & 4 & 62,94 & 39 & 221,8 & 20 & 39,08 & 33 & 20,53 & 8 & 657 & 24 \\
\hline \pm SD & $\pm 6,24$ & & $\pm 17,79$ & & $\pm 0,08$ & & $\pm 30,20$ & & $\pm 98,17$ & & $\pm 30,91$ & & $\pm 11,86$ & & $\pm 23,67$ & & $\pm 5,28$ & & $\pm 155,03$ & \\
\hline & $O D=1,02-$ & & $O D=0,39-$ & & $O D=1,17$ & & $O D=1,34-$ & & $O D=0.99$ & & $O D=0,97$ & & $\begin{array}{c}O D=0 \\
74-\end{array}$ & & $O D=0,59$ & & $\begin{array}{c}O D=0 \\
87\end{array}$ & & $\begin{array}{c}O D=0 \\
86-\end{array}$ & \\
\hline & $p=0,964$ & & $p=0,091$ & & $p=0,419$ & & $p=0,351$ & & $p=0,625$ & & $p=0,567$ & & $p=0,289$ & & $p=0,126$ & & $p=0,498$ & & $p=0,417$ & \\
\hline No (A) & 27,66 & 53 & 107,8 & 57 & 1,30 & 23 & 388 & 11 & 573 & 57 & 36,33 & 13 & 159,0 & 41 & 14,13 & 28 & 8,29 & 53 & 345 & 34 \\
\hline \pm SD & $\pm 8,25$ & & $\pm 25,53$ & & $\pm 0,08$ & & $\pm 22,85$ & & $\pm 179,55$ & & $\pm 7,48$ & & $\pm 27,42$ & & $\pm 3,78$ & & $\pm 2,64$ & & $\pm 89,35$ & \\
\hline No (B) & 48,58 & 9 & 177,8 & 5 & 1,49 & 39 & 474 & 51 & 1533 & 5 & 60,07 & 49 & 215,6 & 21 & 34,57 & 34 & 23,85 & 9 & 735 & 28 \\
\hline \pm SD & $\pm 7,87$ & & $\pm 30,74$ & & $\pm 0,09$ & & $\pm 33,59$ & & $\pm 659,11$ & & $\pm 11,31$ & & $\pm 11,26$ & & $\pm 15,67$ & & $\pm 1,91$ & & $\pm 187,64$ & \\
\hline & & & & & & & & & & & ections & & & & & & & & & \\
\hline Yes (A) & 27,11 & 39 & 109,4 & 40 & 1,29 & 17 & 378 & 6 & 622 & 43 & 35,86 & 12 & 150,4 & 30 & 12,42 & 17 & 8,07 & 37 & 317 & 25 \\
\hline \pm SD & $\pm 5,91$ & & $\pm 20,77$ & & $\pm 0,08$ & & $\pm 49,27$ & & $\pm 190,81$ & & $\pm 4,64$ & & $\pm 30,52$ & & $\pm 3,71$ & & $\pm 2,99$ & & $\pm 87,29$ & \\
\hline Yes (B) & 46,92 & 5 & 164,0 & 4 & 1,50 & 27 & 461 & 38 & 1251 & 1 & 60,40 & 32 & 225,8 & 14 & 36,95 & 27 & 17,97 & 7 & 659 & 19 \\
\hline \pm SD & $\pm 5,19$ & & $\pm 11,53$ & & $\pm 0,07$ & & $\pm 32,08$ & & & & $\pm 11,02$ & & $\pm 6,76$ & & $\pm 15,78$ & & $\pm 1,62$ & & $\pm 174,08$ & \\
\hline & $O D=1,53-$ & & $O D=1,75$ & & $O D=0,99$ & & $O D=0,50-$ & & $O D=5,83-$ & & $O D=1,91-$ & & $\begin{array}{c}O D=1, \\
45\end{array}$ & & $O D=0,93-$ & & $\begin{array}{c}O D=0 \\
93-\end{array}$ & & $\begin{array}{c}O D=1 \\
28-\end{array}$ & \\
\hline & $p=0,325$ & & $p=0,274$ & & $p=0,573$ & & $p=0,139$ & & $p=0,065$ & & $p=0,127$ & & $p=0,241$ & & $p=0,510$ & & $p=0,546$ & & $p=0,333$ & \\
\hline No (A) & 28,56 & 56 & 112,5 & 57 & 1,30 & 26 & 387 & 16 & 584 & 59 & 36,50 & 11 & 162,1 & 40 & 14,23 & 27 & 8,04 & 57 & 342 & 34 \\
\hline$\pm \mathrm{SD}$ & $\pm 8,48$ & & $\pm 24,62$ & & $\pm 0,08$ & & $\pm 23,96$ & & $\pm 184,72$ & & $\pm 7,97$ & & $\pm 25,43$ & & $\pm 3,72$ & & $\pm 2,70$ & & $\pm 86,50$ & \\
\hline No (B) & 50,57 & 11 & 176,1 & 10 & 1,51 & 41 & 469 & 51 & 1339 & 8 & 61,66 & 56 & 215,9 & 27 & 36,58 & 40 & 25,30 & 10 & 727 & 33 \\
\hline \pm SD & $\pm 7,68$ & & $\pm 29,90$ & & $\pm 0,09$ & & $\pm 33,30$ & & $\pm 526,32$ & & $\pm 25,18$ & & $\pm 12,05$ & & $\pm 22,22$ & & $\pm 2,85$ & & $\pm 178,36$ & \\
\hline & & & & & & & & & & Mus & e Injuries & & & & & & & & & \\
\hline Yes (A) & 27,50 & 74 & 110,7 & 72 & 1,31 & 30 & 386 & 18 & 594 & 76 & 36,45 & 13 & 160,9 & 49 & 14,29 & 28 & 7,86 & 68 & 338 & 41 \\
\hline \pm SD & $\pm 7,85$ & & $\pm 22,53$ & & $\pm 0,06$ & & $\pm 32,62$ & & $\pm 190,72$ & & $\pm 7,01$ & & $\pm 26,67$ & & $\pm 3,86$ & & $\pm 2,80$ & & $\pm 86,09$ & \\
\hline Yes (B) & 46,31 & 7 & 167,3 & 9 & 1,50 & 51 & 468 & 63 & 1178 & 5 & 63,24 & 68 & 218,3 & 32 & 32,81 & 53 & 17,97 & 13 & 697 & 40 \\
\hline \pm SD & $\pm 6,71$ & & $\pm 16,36$ & & $\pm 0,09$ & & $\pm 34,71$ & & $\pm 90,85$ & & $\pm 24,33$ & & $\pm 12,47$ & & $\pm 13,43$ & & $\pm 1,62$ & & $\pm 165,13$ & \\
\hline & $O D=4,53-$ & & $O D=1,60-$ & & $O D=0,77$ & & $O D=1,86-$ & & $O D=2,34-$ & & $O D=0,38$ & & $\begin{array}{c}O D=0, \\
66-\end{array}$ & & $O D=0,46-$ & & $\begin{array}{c}O D=0 \\
80-\end{array}$ & & $\begin{array}{c}O D=0 \\
68-\end{array}$ & \\
\hline & $p=0,007$ & & $p=0,312$ & & $p=0,348$ & & $p=0,223$ & & $p=0,197$ & & $p=0,045$ & & $p=0,244$ & & $p=0,058$ & & $p=0,491$ & & $p=0,253$ & \\
\hline No (A) & 29,59 & 21 & 112,7 & 25 & 1,25 & 13 & 376 & 4 & 618 & 26 & 35,80 & 10 & 148,1 & 21 & 12,19 & 16 & 8,55 & 26 & 316 & 18 \\
\hline \pm SD & $\pm 6,17$ & & $\pm 24,89$ & & $\pm 0,10$ & & $\pm 29,97$ & & $\pm 179,45$ & & $\pm 5,59$ & & $\pm 30,03$ & & $\pm 3,34$ & & $\pm 2,81$ & & $\pm 89,35$ & \\
\hline No (B) & 51,86 & 9 & 193,5 & 5 & 1,50 & 7 & 460 & 26 & 1478 & 4 & 55,73 & 20 & 218,6 & 9 & 47,16 & 14 & 25,30 & 4 & 721 & 12 \\
\hline \pm SD & $\pm 6,64$ & & $\pm 40,31$ & & $\pm 0,05$ & & $\pm 28,52$ & & $\pm 698,37$ & & $\pm 6,94$ & & $\pm 10,92$ & & $\pm 29,29$ & & $\pm 2,85$ & & $\pm 211,13$ & \\
\hline
\end{tabular}

Table 3: Comparison of micronutrients less than the middle of standard against more than the middle of standard in terms of the symptoms. 


\section{Discussion}

Our results indicate that in top athletes - regardless of which sport - a deficiency of immunologically (vitamin D and selenium) and mitochondrially essential (intracellular iron and magnesium as well as coenzyme Q10) micronutrients is widely spread in $27.1 \%-69.4 \%$. Young elite athletes are in these micronutrients in $35.7 \%-79.1 \%$ significantly worse supplied than adults athletes with $21.4 \%-63.2 \%$ and thus have a 2 - 2.72 times higher risk of suffering from deficiency of these micronutrients than adults. A significant 3-fold higher risk for muscular injuries in adolescents $(86 \%$ in adolescents vs $67 \%$ in adults $-\mathrm{OR}=3.00 ; 95 \% \mathrm{CI}: 1.50-4.50, \mathrm{p}=0.022$ ) is the consequence.

Only $2.2-10 \%$ of the study participants have a deficiency in the B vitamin supply, where the young people $(3-16.2 \%)$ are affected more frequently than the adults $(0-10.9 \%)$ - with the exception of folic acid (vitamin B9): here the young people ( $8.3 \%$ vs. $10.9 \%$ ) are minimally better supplied. The abundance of the results in comparison to the literature makes a discussion of each individual micronutrient meaningful.

\section{Vitamin D}

In our study, the prevalence rate of inadequate vitamin D supply $(51.4 \%)$ correlated closely with the metaanalysis recently published by Farrokhyar et al [46], in which $56 \%$ of the athletes examined worldwide showed inadequate vitamin D supply $(<30 \mathrm{ng} / \mathrm{ml})$. The prevalence of inadequate vitamin D supply in other studies $[25, \mathbf{2 6}, \mathbf{2 8}, \mathbf{3 1}]$ ranged from $51 \%$ $91,3 \%$ of the athletes, with the average vitamin D value of the athletes $(\mathrm{N}=20969)$ in these studies ranging from 11,62 $\pm 9,24$ $\mathrm{ng} / \mathrm{ml}-38,45 \pm 16,65 \mathrm{ng} / \mathrm{ml}[\mathbf{2 3 - 2 6}, \mathbf{2 8 - 3 3}]$. The average vitamin $\mathrm{D}$ value of the study with most participants $(\mathrm{N}=18883)$ correlated with $29 \pm 8 \mathrm{ng} / \mathrm{ml} \mathrm{[33]} \mathrm{and} \mathrm{the} \mathrm{value} \mathrm{of} \mathrm{the} \mathrm{Swiss}$ Olympic athletes $(\mathrm{N}=651)$ with $30.32 \pm 9.24 \mathrm{ng} / \mathrm{ml}$ [25] closely correlated with the average value in our study $(\mathrm{N}=111): 31.06 \pm 10.61 \mathrm{ng} / \mathrm{ml}$. Adolescents, with an average of $25.71 \pm 9.58 \mathrm{ng} / \mathrm{ml}$, have a significantly worse vitamin D supply than national A players $(35.78 \pm 12.35 \mathrm{ng} / \mathrm{ml}$ $\mathrm{p}=0.007)$. In our study, the proportion of adolescent athletes within inadequate vitamin D levels $(<30 \mathrm{ng} / \mathrm{ml})$ was $64.4 \%$, significantly worse compared to adults $(42.4 \%$ - OR $=2.46$ $\mathrm{p}<0.023)$. These results confirm the observations of Quadri et al [25]: $65 \%(234 / 360)$ of Swiss athletes $<18$ years of age had a vitamin D supply < 30ng/ml compared to $34 \%(99 / 192)$ of athletes $>18$ years of age $(\mathrm{OR}=3.6)$, whereby the risk of suffering a vitamin D deficiency $(<20 \mathrm{ng} / \mathrm{ml})$ in adolescents $<$ 18 years of age compared to adults in this study is 5.18 times higher $(95 \%$ CI $3.00-8.95, \mathrm{p}<0.0001)$. The reason for the significantly worse vitamin D supply in adolescents maybe on the one hand the high consumption during growth age, on the other hand the better average supply in A-national players may be caused by a more conscious diet (e.g. more fish) but also by a targeted, association-controlled vitamin D substitution.

The striking feature of our study is the large number of muscle injuries among top athletes (73\% of all athletes), whereby the U16 and U18 national players also suffered much more from muscular injuries $(83 \%$ of the $\mathrm{U} 16$ and $87 \%$ of the
U18 athletes). Among athletes with muscular injuries, the vitamin D level of $29.13 \pm 9.38 \mathrm{ng} / \mathrm{ml}$ was lower (=inadequate vitamin D supply) than among athletes without muscular injuries $(36.27 \pm 12.09 \mathrm{ng} / \mathrm{ml})$. Quadri et al [25] found similar results. According to this study, $62.2 \%$ of Swiss Olympic athletes complained of muscular pain. In a comparison of the prevalence of muscular injuries in athletes with vitamin $D$ values > $40 \mathrm{ng} / \mathrm{ml}$ to athletes with vitamin $\mathrm{D}$ values $<40$ $\mathrm{ng} / \mathrm{ml}$, the probability of muscular injuries in athletes with vitamin $\mathrm{D}$ values $<40 \mathrm{ng} / \mathrm{ml}$ is significantly higher than in athletes with vitamin D values $>40 \mathrm{ng} / \mathrm{ml}(\mathrm{OR}=4.53, \mathrm{KI} 95 \%$ $=[3.43 ; 5.63], p=0,007)$. In this context, the influence of adequate vitamin D concentrations $(>40 \mathrm{ng} / \mathrm{ml}$ ) on the TNF $\alpha$ concentration seems to play a decisive role. Willis et al [32] were able to demonstrate a significant inverse correlation between vitamin D and TNF concentrations in 19 healthy athletes, with vitamin D levels > $40 \mathrm{ng} / \mathrm{ml}$ being the target. TNF- $\alpha$ leads in the acute phase to an increased cellular calcium release and thus to muscle stiffness and rupture while in the intermediate phase the cellular inflammation parameter $\mathrm{Nf} \kappa \mathrm{B}$ increases resulting in an increased macrophage reaction resulting in muscular inflammation and injury [47]. Can vitamin $\mathrm{D}$ values $>70 \mathrm{ng} / \mathrm{ml}$ prevent muscular injuries by lowering the $\mathrm{TNF} \alpha$ concentration in competitive sports (Figure 2)?.

A placebo-controlled randomized therapy study in athletes with vitamin $D$ deficiency $(\mathrm{N}=40: 18 \pm 10 \mathrm{ng} / \mathrm{ml})$ in which Owens et al [24] achieved a significant increase in vitamin D levels in the therapy group $(\mathrm{N}=20: 46 \pm 12.4 \mathrm{ng} / \mathrm{ml}-\mathrm{p}<0.005)$ by daily substitution of $4000 \mathrm{IU}$ OH-VitaminD3 over 6 weeks and observed a significantly faster recovery of these vitamin D levels in the therapy group compared to the placebo group in muscular injuries. Close et al (23) were able to confirm the improvement of muscle strength through vitamin D substitution in their therapy study. In this placebo-controlled randomized trial, a significant increase of vitamin D > $40 \mathrm{ng} / \mathrm{ml}$ was achieved in the verum group by daily supplementation with 5000 IU OHVitaminD3 over 8 weeks. At vitamin D levels > $40 \mathrm{ng} / \mathrm{ml}$, a significant increase in the $10 \mathrm{~m}$ and $30 \mathrm{~m}$ sprint force and bounce could be observed in the verum group compared to the placebo group [23].

Correlations between vitamin D values and sleep disorders, fatigue and infections showed no significance, although athletes with vitamin D values $<40 \mathrm{ng} / \mathrm{ml}$ showed a 1.53 -fold higher risk of contracting infections than athletes with vitamin D values $>40 \mathrm{ng} / \mathrm{ml}$. The Third National Health and Nutrition Examination Survey [33] seems to confirm this observation, as this study $(\mathrm{N}=18883)$ showed a significant inverse correlation between vitamin $\mathrm{D}$ concentration and upper respiratory tract infections (URTI) (participants with vitamin D levels $<10$ $\mathrm{ng} / \mathrm{ml}$ had URTI in up to $30 \%$ of cases). On the international level, the daily dose of 4000 - 5000 IU $250 H V i t a m i n D 3$ achieves a target value for vitamin $\mathrm{D}>40 \mathrm{ng} / \mathrm{ml}$ and is recommended, similar to our results (Table 4). Further therapy studies with doses between 10,000 - 30,000 IU 25OHVitaminD3 must show whether target values for vitamin D in the upper normal range $(70-80 \mathrm{ng} / \mathrm{ml})$ can lead to better results in the prevention of muscular injuries or recurrent infections. 


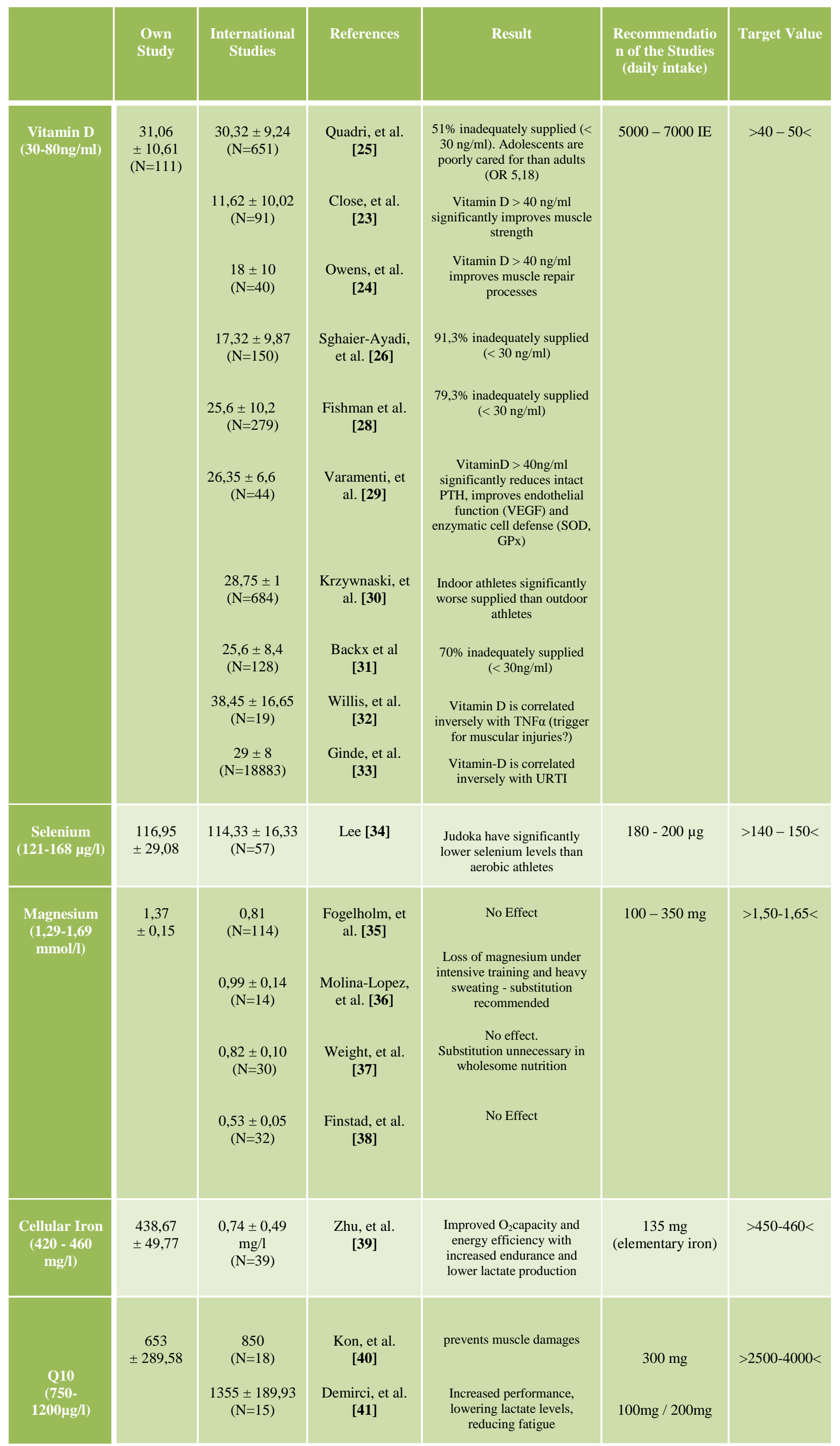




\begin{tabular}{|c|c|c|c|c|c|c|}
\hline & & $\begin{array}{c}\text { k.a. } \\
(\mathrm{N}=100)\end{array}$ & $\begin{array}{l}\text { Alf, et al. } \\
\text { [42] }\end{array}$ & $\begin{array}{l}\text { Increased performance and } \\
\text { maximum power }\end{array}$ & $300 \mathrm{mg}$ & \\
\hline $\begin{array}{l}\text { Vitamin B1 } \\
(30-90 \mu g / 1)\end{array}$ & $\begin{array}{c}54,82 \\
\pm 21,62\end{array}$ & $\begin{array}{c}\text { k.a. } \\
(\mathrm{N}=27) \\
\\
\\
23,53 \pm 4,07 \\
(\mathrm{~N}=9)\end{array}$ & $\begin{array}{l}\text { Bautista- } \\
\text { Hernandez, et } \\
\text { al. [45] } \\
\text { Choi, et al. } \\
\text { [43] }\end{array}$ & $\begin{array}{l}\text { Lower lactate levels and } \\
\text { larger VO2max under } \\
\text { performance } \\
\text { Lower lactate levels under } \\
\text { performance } \\
\text { fatigue decreases under } \\
\text { substitution }\end{array}$ & $\begin{array}{c}1 \mathrm{mg} / \mathrm{kg} \mathrm{KG} \\
\text { as infusion } \\
30 \mathrm{mg}\end{array}$ & $>80-90<$ \\
\hline $\begin{array}{c}\text { Vitamin B2 } \\
(137-270 \mu \mathrm{g} / \mathrm{l})\end{array}$ & $\begin{array}{l}170,68 \\
\pm 36,06\end{array}$ & $\begin{array}{c}10,6 \pm 1 \\
(\mathrm{~N}=30)\end{array}$ & $\begin{array}{c}\text { Weight, et al. } \\
\text { [37] }\end{array}$ & $\begin{array}{l}\text { No Effect } \\
\text { Substitution unnecessary in } \\
\text { wholesome nutrition }\end{array}$ & $?$ & $?$ \\
\hline$\frac{\text { Vitamin B6 }}{(5-30 \mu \mathrm{g} / \mathrm{I})}$ & $\begin{array}{c}25,13 \\
\pm 15,24\end{array}$ & $\begin{array}{l}19,52 \pm 20,76 \\
(\mathrm{~N}=30) \\
\\
10,38 \pm 3,46 \\
(\mathrm{~N}=264)\end{array}$ & $\begin{array}{l}\text { Weight, et al. } \\
\qquad \text { [37] } \\
\text { Manore [44] }\end{array}$ & $\begin{array}{l}\text { No Effect } \\
\text { Substitution unnecessary in } \\
\text { wholesome nutrition } \\
\text { High intens training leads to a } \\
\text { B6 deficiency in 50\% }\end{array}$ & $1,5 \mathrm{mg}$ & $?$ \\
\hline $\begin{array}{c}\text { Folic Acid } \\
(4,6-18,7 \\
n g / m l)\end{array}$ & $\begin{array}{c}8,87 \\
\pm 4,35\end{array}$ & $\begin{array}{c}5,5 \pm 2,6 \\
(\mathrm{~N}=30)\end{array}$ & $\begin{array}{c}\text { Weight, et al. } \\
\text { [37] }\end{array}$ & $\begin{array}{l}\text { No Effect } \\
\text { Substitution unnecessary in } \\
\text { wholesome nutrition }\end{array}$ & $800-1200 \mu \mathrm{g}$ & $>15<$ \\
\hline $\begin{array}{c}\text { Vitamin B12 } \\
(200-950 \\
\mathrm{pg} / \mathrm{ml})\end{array}$ & $\begin{array}{c}465,72 \\
\pm 219,57\end{array}$ & $\begin{array}{c}470,3 \pm 160,9 \\
(\mathrm{~N}=30)\end{array}$ & $\begin{array}{l}\text { Weight, et al. } \\
\text { [37] }\end{array}$ & $\begin{array}{l}\text { No Effect } \\
\text { Substitution unnecessary in } \\
\text { wholesome nutrition }\end{array}$ & $1000 \mu \mathrm{g}$ & $>800<$ \\
\hline
\end{tabular}

Table 4: Micronutrients in international studies

\section{Selenium}

In our study, the prevalence of inadequate cellular selenium supply $(<121 \mu \mathrm{g} / \mathrm{l}, 57 \%)$ is unique in the international literature. Solely the average selenium value of the athletes in our study of $116.95 \pm 29.08 \mu \mathrm{g} / 1$ is confirmed in the publication of Lee [34] with $114.33 \pm 16.33 \mu \mathrm{g} / \mathrm{l}(\mathrm{N}=57)$, whereby an aerobically training athletes (judoka) were significantly worse supplied with selenium than aerobically training athletes $(107 \pm 13 \mu \mathrm{g} / \mathrm{l}$ vs. $122 \pm 16 \mu \mathrm{g} / \mathrm{l}-\mathrm{p}<0.05)$.

With an average of $106.47 \pm 29.71 \mu \mathrm{g} / \mathrm{l}, \mathrm{U} 16 / 18$ national players have a lower intracellular selenium supply than first squad players $(123.23 \pm 32.90 \mu \mathrm{g} / \mathrm{l})$. In our study, with $66.7 \%$, the proportion of young athletes inadequately supplied with cellular selenium $(<121 \mu \mathrm{g} / \mathrm{l})$ is higher than in adults. The reason for the significantly lower intracellular selenium supply in adolescents may also be the higher requirement during growth age (especially for maintaining thyroid function). It is not known whether A-national players eat more consciously in relation to this parameter. However, they were provided with an electrolyte drink containing selenium as a dietary supplement on the average of 200-250 training days per year. In our study, it was not possible to prove any connections between inadequate selenium supply in contrast to sufficient cellular selenium supply in comparison to fatigue, sleep disorders, infections and muscular injuries. Also in the international literature, therapy studies (daily substitution of 180 - $200 \mu \mathrm{g}$ selenomethionine over 3 - 10 weeks) could not prove any effects, especially on an increase in performance $[48,49]$. Exclusively Tessier et al [50] were able to demonstrate a significant increase in glutathione peroxidase activity (enzymatic protection against oxidative stress) in selenium-substituted athletes against placebo under sports stress, whereby no cellular selenium values were reported.

\section{Intracellular Magnesium}

The prevalence of inadequate cellular magnesium supply $(<129 \mathrm{mmol} / \mathrm{l})$ in our study $(27.1 \%)$ is not reflected in the international literature. In the Casoni study [52], only the average cellular magnesium value of $1.37 \pm 0.15 \mathrm{mmol} / \mathrm{l}$ of the athletes in our studies was confirmed with $1.30 \mathrm{mmol} / \mathrm{l}$ $(\mathrm{N}=41)$, whereas the cross-country athlete $(25 \mathrm{~km})$ were significantly worse supplied with magnesium than the control group $(1.3 \mathrm{mmol} / \mathrm{l}$ vs. $1.8 \mathrm{mmol} / \mathrm{l}-\mathrm{p}<0.01)$.

Prospective U16/18 athletes have a lower cellular magnesium supply with an average of $1.31 \pm 0.12 \mathrm{mmol} / \mathrm{l}$ than national a players $(1.42 \pm 0.15 \mathrm{mmol} / \mathrm{l})$. The proportion of young athletes supplied with cellular magnesium in adequately $(<1.29 \mathrm{mmol} / \mathrm{l})$ in our study is $35.7 \%$ higher than compared to adults $(21.4 \%-$ OR $=2.04-\mathrm{p}<0.188)$, too. Magnesium is involved in many enzymatic detoxification and protein metabolism processes and is a central cofactor for all neurotransmitters. Circumstances could be a reason for the poorer cellular magnesium supply in adolescents with a higher need for growth and simultaneous school stress. Conversely, A squad players are specifically substituted with magnesium supplements of up to $400 \mathrm{mg}$ per day. Statistically significant correlation between inadequate and sufficient cellular magnesium supply with regard to fatigue, sleep disorders, infections and muscular injuries could not be proven in our study. However, in the international literature, placebocontrolled therapy studies (daily substitution of 100 - $350 \mathrm{mg}$ magnesium over 4 - 12 weeks) have shown significant effects (reduction of lactate production, faster recovery and increase muscle strength and training performance) $[\mathbf{3 6}, \mathbf{5 2}, \mathbf{5 3}]$.

\section{Intracellular Iron}

The examination of intracellular iron values in competitive athletes has not yet been described in the literature. Worldwide studies define the iron metabolism via the iron storage ferritin 
$[54,55,58,59]$ or via the syndromes non-anaemic iron deficiency (NAID) or anaemic iron deficiency (AID) [56, 57, $59,60]$. According to our study, NAID would correspond to an adequate intracellular iron value ( $>420 \mathrm{mg} / \mathrm{l}$ ) and AID to an intracellular iron deficiency $(<420 \mathrm{mg} / \mathrm{l})$. Considering the physiological iron distribution, ferritin represents only $25 \%$ of the iron volume (1000mg of $4000 \mathrm{mg}$ total storage) and plasma or serum iron only $1 \%$ (5mg of $4000 \mathrm{mg}$ ) (see Figure 3). However, the intraerythrocytic (cellular) iron represents $60 \%$ of the total body iron $(2400 \mathrm{mg}$ of $4000 \mathrm{mg}$ ) and $100 \%$ of the cellular oxygen transport (see Figure 3).

Currently, the prevalence of inadequate cellular iron supply $(<420 \mathrm{mg} / \mathrm{l})$ in our study (34.3\%) is not described in the international literature. Compatible with this is AID, which is observed in studies just less than $3 \%$ of the study participants $[\mathbf{5 6}, \mathbf{5 7}, \mathbf{5 9}, \mathbf{6 0}]$. A ferritin-based study reported the prevalence of iron storage deficiency at $37 \%$, with ferritin deficiency $13 \%$ higher in female subjects then in male subjects $[55,57,59,60]$. In our study, female athletes also had inadequate and significantly lower cellular iron levels than male athletes $(403.60 \pm 35.72 \mathrm{mg} / \mathrm{l}$ vs. $477.03 \pm 31.10 \mathrm{mg} / \mathrm{l}$ $\mathrm{p}<0.0005)$.
With an average of $407.13 \pm 43.09 \mathrm{mg} / \mathrm{l}, \mathrm{U} 16 / \mathrm{U} 18$ national players have a significantly worse supply of cellular iron than A national players or adults $(460.29 \pm 36.69 \mathrm{mg} / \mathrm{l}-\mathrm{p}=0.018$ or $452.29 \pm 50.67 \mathrm{mg} / \mathrm{l}-\mathrm{p}=0.026)$. Also the proportion of young athletes inadequately supplied with cellular iron $(<420 \mathrm{mg} / \mathrm{l})$ in our study is with $46.7 \%$ significantly higher than compared to adults $(24.3 \%-\mathrm{OR}=2.72-\mathrm{p}<0.055)$. Iron is the central atom of the haem complex and necessary for sufficient oxygen supply to the cells and thus for optimal function of the mitochondrial respiratory chain. In adolescents, a reason for the poor cellular iron supply may be the diet (high proportion of carbohydrates and low proportion of meat). On the other hand, in the elderly area of the association, iron dose of up to $200 \mathrm{mg}$ per day are specifically substituted. Statistically significant correlations between an inadequate iron supply compared to a sufficient cellular iron supply in relation to the parameters fatigue, sleep disorders, infections and muscular injuries were not detectable in our study. In a placebo controlled therapy study ( $\mathrm{N}=37$ with NAID: daily substitution of $135 \mathrm{mg}$ iron over 8 weeks), Zhu et al [39] achieved significant effects in terms of improved $\mathrm{O} 2$ capacity and energy efficiency with increase endurance and reduce lactate production.

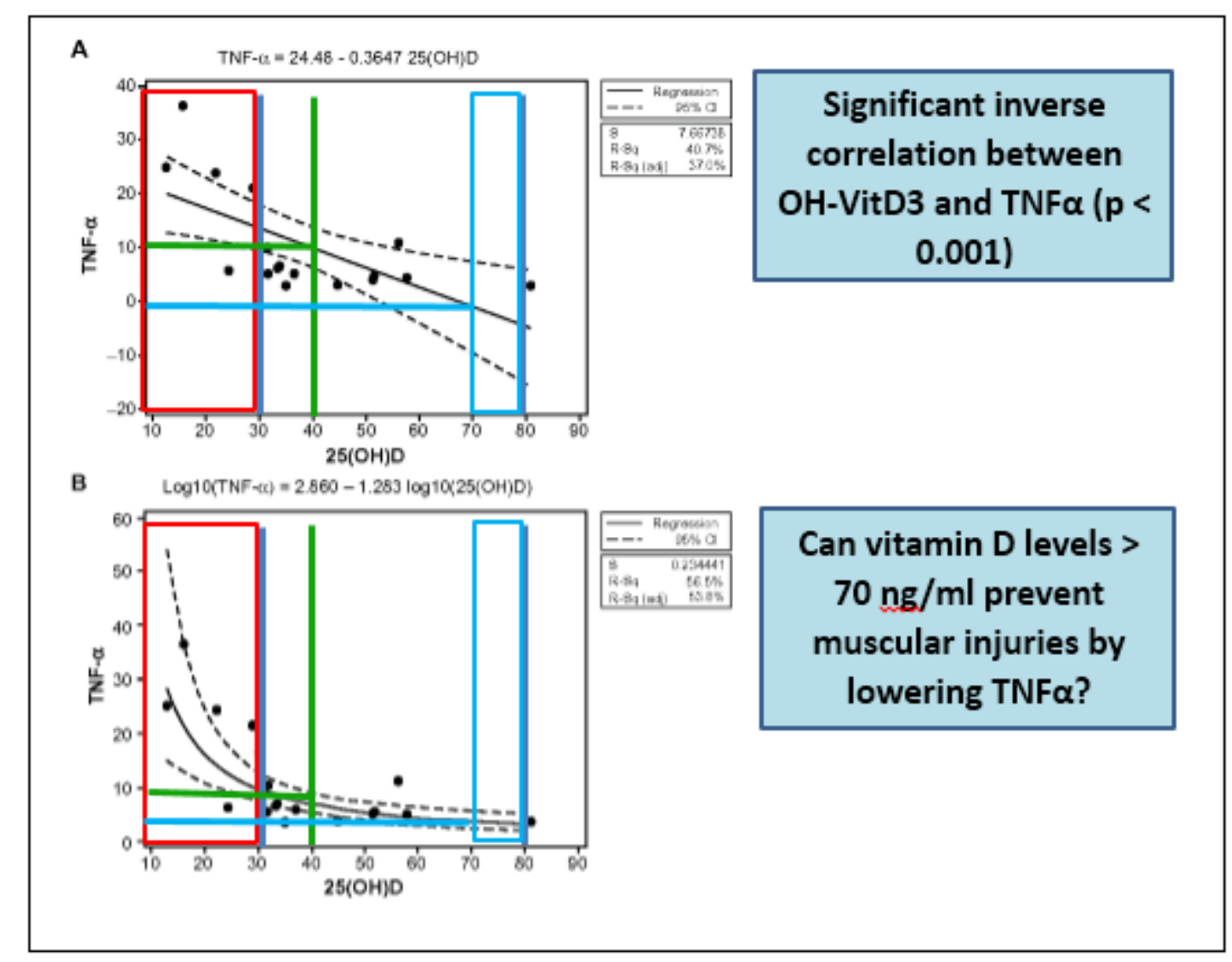

Athletes Value Worldwide

\section{Target Value}

Worldwide $40 \mathrm{ng} / \mathrm{ml}$
Optimal Value 70 - $80 \mathrm{ng} / \mathrm{ml}$
Standard Range $30-80 \mathrm{ng} / \mathrm{ml}$

Figure 2: inverse correlation TNF $\alpha$ and OH-VitD3 according to Willis et al. (32) modified according to Erpenbach et al. [61] (unpublished data). 


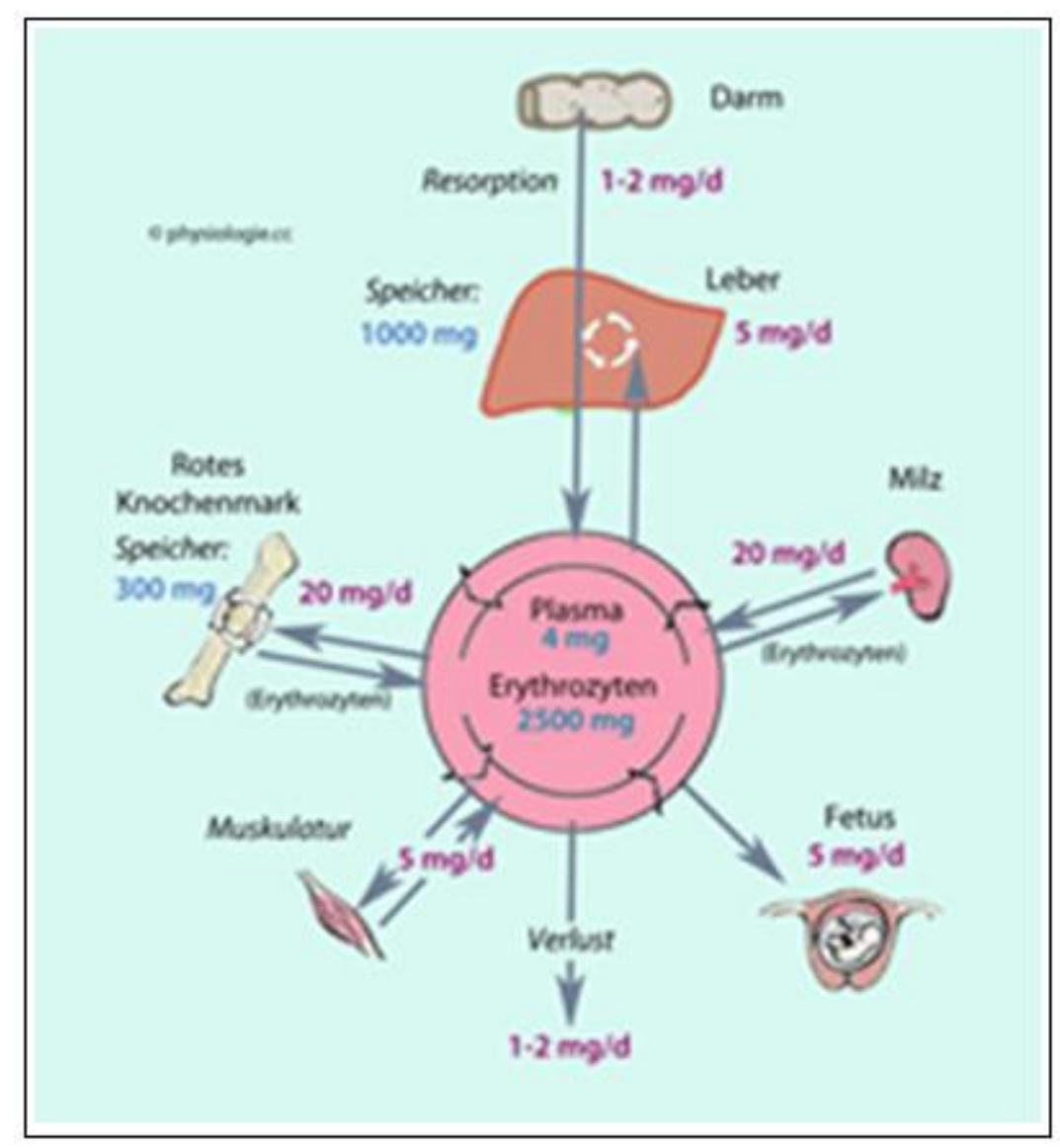

Figure 3: Iron distribution in the body (www.physiologie.cc/ XI.6.html).

\section{CoenzymeQ10}

The prevalence of an inadequate Q10 supply $(<750 \mu \mathrm{g} / \mathrm{l})$ in our study with $69.4 \%$ cannot be found in the literature. The average Q10 values of international studies $(\mathrm{N}=33)$ with 850 $\mu \mathrm{g} / \mathrm{l}[\mathbf{4 0}]$ and $1355 \pm 189.93 \mu \mathrm{g} / \mathrm{l}$ [41] show large deviations from the mean value of our study ( $\mathrm{N}=111): 653 \pm 289.58 \mu \mathrm{g} / \mathrm{l}$. With an average of $579.25 \pm 233.63 \mu \mathrm{g} / \mathrm{l}, \mathrm{U} 16 / \mathrm{U} 18$ national players have a worse supply of Q10 than A national players $(751.71 \pm 441.80 \mu \mathrm{g} / \mathrm{l})$. With $79.1 \%$ in our study, the proportion of young athletes with inadequately Q10 supply (< $750 \mu \mathrm{g} / \mathrm{l})$ is higher than that of adults $(63.2 \%-\mathrm{OR}=2.20$ $\mathrm{p}<0.078)$. There is no comparison in the literature. The reason for the significantly poorer Q10 supply in adolescents may be the increased mitochondrial energy and proliferation requirements during growth. The Q10 vitamin plays the central role in the mitochondrial respiratory chain for the generation of ATP energy and for protein synthesis in the citrate cycle. As already mentioned, the high number of muscle injuries in top athletes is very noticeable in our study (73\% of all athletes - U16/ U18 national players with $83 \%$ and adolescents under 18 years of age with $87 \%$ significantly more frequently). For athletes with muscle injuries the Q10 value of $623.31 \pm 226.31 \mu \mathrm{g} / \mathrm{l}$ was lower (=inadequate Q10 supply) than for athletes without muscular injuries $(732.93 \pm 408.19$ $\mu \mathrm{g} / \mathrm{l})$. Comparative data for international studies are not available. Comparing the prevalence of muscular injury and infection in athletes with Q10 values> $750 \mu \mathrm{g} / \mathrm{l}$ with Q10 values $<750 \mu \mathrm{g} / \mathrm{l}$, the probability of muscular injury or infection is higher for Q10 values< $750 \mu \mathrm{g} / \mathrm{l}$ than for athletes with $\mathrm{Q} 10$ values $>750 \mathrm{n} \mu \mathrm{g} / \mathrm{l}(\mathrm{OR}=2.34$ - $\mathrm{p}<0,197$ and $\mathrm{OR}=5,83$ $\mathrm{p}<0.065$ ), respectively. Enhanced oxidative phosphorylation in the mitochondrial respiratory chain (Q10-dependent) and a resulting decrease in muscular lactate production (longer optimized aerobic stress phase) lead to a decrease in CK values (muscular enzymes in micro injuries) and seem to explain our observations [41, 41]. In a placebo-controlled therapy study (N=18 Kendo athletes - daily 300mg Q10 for 3 weeks) Kon et al (40) were able to achieve a significant increase in the Q10 value compared to placebo athletes (verum: $850 \mu \mathrm{g} / \mathrm{l}$ to 4000 $\mu \mathrm{g} / \mathrm{l}$ vs placebo: $855 \mu \mathrm{g} / \mathrm{l}$ to $865 \mu \mathrm{g} / \mathrm{l}-\mathrm{p}<0.01$ ). A significant reduction in CK activity was observed during the training camp (verum: from $255 \mathrm{U} / 1$ to $830 \mathrm{U} / 1$ vs placebo: $260 \mathrm{U} / 1$ to
$1500 \mathrm{U} / \mathrm{l}$ - $\mathrm{p}<0.05$ ). Demirci et al (41) confirm these results in their placebo-controlled study. They divided 15 athletes into 3 groups (placebo - daily 100mg Q10 - daily 200mg Q10 over 1 week training camp). During the one-week therapy phase, the Q10 value in the verum groups increased significantly with substitution compared to the placebo group (100mg: $1570 \pm$ $220 \mu \mathrm{g} / \mathrm{l}$ to $2240 \pm 730 \mu \mathrm{g} / \mathrm{l}$ vs $200 \mathrm{mg}: 2120 \pm 320 \mu \mathrm{g} / \mathrm{l}$ to 3800 $\pm 540 \mu \mathrm{g} / \mathrm{l}$ vs placebo: $1570 \pm 560 \mu \mathrm{g} / \mathrm{l}$ to $1500 \pm 270 \mu \mathrm{g} / \mathrm{l}-$ $\mathrm{p}<0.01)(41)$. According to this optimization, a significant reduction in CK activity could be demonstrated in the verum groups compared to the placebo group (100mg: $131.4 \pm 8.01$ $\mathrm{U} / 1$ to $583.6 \pm 9.86 \mathrm{U} / \mathrm{l}$ vs $200 \mathrm{mg}$ : $129 \pm 2.01 \mathrm{U} / \mathrm{l}$ to $499.6 \pm$ $13.3 \mathrm{U} / \mathrm{l}$ vs placebo: $135 \pm 15.6 \mathrm{U} / \mathrm{l}$ to $648.4 \pm 19.5 \mathrm{U} / \mathrm{l}-$ $\mathrm{p}<0.001$ ). Furthermore, the mitochondrial function (lactate dehydrogenase LDH) could be fully maintained under Q10 substitution (100mg: $146.2 \pm 13.7 \mathrm{U} / 1$ to $271.4 \pm 11.2 \mathrm{U} / \mathrm{l}$ vs 200mg: $118 \pm 12.9 \mathrm{U} / \mathrm{l}$ to $240.9 \pm 9.24 \mathrm{U} / \mathrm{l}$ vs placebo: $151 \pm$ $12.9 \mathrm{U} / \mathrm{l}$ to $442.6 \pm 32.4 \mathrm{U} / \mathrm{l}($ norm $<250 \mathrm{U} / \mathrm{l})$ - $\mathrm{p}<0.001$ ). While maintaining optimal mitochondrial oxidative phosphorylation under Q10 substitution, lactate accumulation during the training camp was significantly lower (100mg: $2.59 \pm 0.26$ $\mathrm{mmol} / \mathrm{l}$ to $1.21 \pm 0.33 \mathrm{mmol} / \mathrm{l}$ vs $200 \mathrm{mg}: 2.37 \pm 0.37 \mathrm{mmol} / \mathrm{l}$ to $0.55 \pm 0.16 \mathrm{mmol} / \mathrm{l}$ vs placebo: $2.15 \pm 0.12$ to $4.40 \pm 0.66$ $\mathrm{mmol} / 1-\mathrm{p}<0.01)[41,61]$. In a placebo- controlled study involving 100 German Olympic athletes (placebo vs. 300mg Q10 daily over 6 weeks), Alf et al (42) demonstrated a significant increase in performance (verum: $11.03 \%$ vs. placebo: $8.54 \%$ - $\mathrm{p}=0.03$ ). Furthermore, it needs to be shown if doses between $300-400 \mathrm{mg}$ of natural Q10 and target values in the range of $2500-4000 \mu \mathrm{g} / \mathrm{l}$ can lead to better results in the prevention of muscular injuries.

\section{Vitamin B1 (Thiamine)}

The prevalence of inadequate thiamine supply $(<30 \mu \mathrm{g} / \mathrm{l})$ in our study (4.4\%) is lower compared to the results of Manore [44] with $12-17 \%$. This may be due to the higher average value $(54.82 \pm 21.62 \mu \mathrm{g} / \mathrm{l})$ for study compared to international studies $(23.53 \pm 4.07 \mu \mathrm{g} / \mathrm{l})$ [43]. Thereby, national players show higher thiamine values (A squad: $53.14 \pm 9.52 \mu \mathrm{g} / \mathrm{l}-$ U16/U18: $54.84 \pm 11.18 \mu \mathrm{g} / \mathrm{l}$ ) compared to non-national players (adults: $49.93 \pm 21.62 \mu \mathrm{g} / \mathrm{l}$ - adolescents< 18 years: $49.93 \pm 17.69 \mu \mathrm{g} / \mathrm{l})$. In our study, the proportion of young 
athletes adequately supplied with thiamine $(<30 \mu \mathrm{g} / \mathrm{l})$ was $7.7 \%$ and more worse compared to adults $(2 \%-\mathrm{OR}=4.25$ $\mathrm{p}<0.186)$. There is no comparison in the literature. The reason for the slightly poorer thiamine supply in adolescents should be a daily significantly increased consumption of carbohydrates (bread, pasta, rice, fruit, and dairy products) compared to the adult athletes of the increased need in the growth phase. In combination with $\alpha$ lipoic acid, thiamine is responsible for the introduction of carbohydrates (via pyruvate modification) into the mitochondrial citrate cycle and thus for amino acid production (regeneration, growth). A correlation between inadequate and sufficient thiamine supply compared to fatigue, sleep disturbance and infections could not be demonstrated in our study. It is currently not possible to explain physiological why low-normal thiamine levels $(35.8 \pm$ $5.59 \mu \mathrm{g} / \mathrm{l})$ can significantly reduce muscle injuries in $62 \%$ $(\mathrm{p}<0.045)$ compared to high-normal levels $(63.24 \pm 24.33$ $\mu \mathrm{g} / \mathrm{l})$, and is the subject of further studies. However, high physical activities (training, competition) lead to a significant decrease in thiamine levels [44]. In placebo-controlled therapy studies, the effect of thiamine substitution (one-time $1 \mathrm{mg} / \mathrm{kg}$ body weight as infusion immediately before exercise or $3 \times 10 \mathrm{mg}$ over 4 weeks) on lactate, $\mathrm{O} 2$ uptake and fatigue was tested [43.45]. Choi et al [43] were able to significantly reduce the lactate level in the verum group by daily substitution of $3 \times 10 \mathrm{mg}$ over 4 weeks $(\mathrm{N}=9)$ under defined ergo meter load (beginning: $0.56 \pm 0.14 \mathrm{mmol} / 1$ vs. $0.56 \pm 0.17 \mathrm{mmol} / 1$ - after 60 minutes load: $2.25 \pm 0.95 \mathrm{mmol} / \mathrm{l}$ vs $4.05 \pm 1.66 \mathrm{mmol} / \mathrm{l}$ after 30 minutes recovery: $1.10 \pm 0.45 \mathrm{mmol} / \mathrm{l}$ vs $1.78 \pm 0.89$ $\mathrm{mmol} / \mathrm{l}-\mathrm{p}<0.05$ ) and exercise fatigue (BORG RPE scale: $12.56 \pm 1.94$ vs $15.11 \pm 2.57-\mathrm{p}<0.05$ ) compared to the placebo group. Bautista-Hernandez et al [45] also achieved a significant reduction in lactate levels (verum vs. placebo before exercise) in their therapy study (single infusion with $1 \mathrm{mg}$ thiamine per kg body weight before treadmill step loading at $\max 16 \mathrm{~km} / \mathrm{h}): 1.92 \pm 0.11 \mathrm{mmol} / \mathrm{l}$ vs $2.18 \pm 0.16 \mathrm{mmol} / \mathrm{l}-$ after loading: $3.34 \pm 0.15 \mathrm{mmol} / \mathrm{l}$ vs $4.56 \pm 0.31 \mathrm{mmol} / \mathrm{l}-$ $\mathrm{p}=0.00001$ ) and a significant increase in $\mathrm{VO} 2 \mathrm{max}$ (verum: $48.2 \pm 1.44 \mathrm{ml} / \mathrm{kg}$. min vs Placebo: $41.9 \pm 1.15 \mathrm{ml} / \mathrm{kg}$. $\mathrm{min}-$ $\mathrm{p}=0.000006)$.

\section{Vitamin B2 (Riboflavin)}

The prevalence of inadequate riboflavin supply $(<137$ $\mu \mathrm{g} / \mathrm{l})$ in our study (8.9\%) is clearly different from the results of Manore [44] with $4-57 \%$. This may be due to the clearly higher riboflavin average value $(170.68 \pm 36.06 \mu \mathrm{g} / \mathrm{l})$ of our study compared to international studies $(10.6 \pm 1 \mu \mathrm{g} / \mathrm{l})$ [37]. Adult athletes have higher riboflavin values (first squad: $177.11 \pm 29.20 \mu \mathrm{g} / \mathrm{l}$ - adults: $178.06 \pm 30.07 \mu \mathrm{g} / \mathrm{l})$ than adolescent athletes (U16/U18: $163.03 \pm 42.29 \mu \mathrm{g} / \mathrm{l}$ adolescents $<18$ years: $156 \pm 43.90 \mu \mathrm{g} / \mathrm{l})$. The proportion of juvenile athletes in our study who had inadequate riboflavin values $(<137 \mu \mathrm{g} / \mathrm{l})$ was $16.2 \%$, significantly worse than that of adults $(3.8 \%-\mathrm{OR}=4.94-\mathrm{p}<0.041)$ There are no comparisons in the literature. The reason for the significantly poorer vitamin B2 supply in adolescents may also be the significantly increased daily intake of carbohydrates (bread, pasta, rice, fruit, dairy products) compared to adult athletes or the increased need during the growth phase. Riboflavin is responsible for the introduction of fatty acids into the mitochondrial citrate cycle and thus for amino acid production (regeneration, growth). Correlations between inadequate and sufficient riboflavin supply compared to fatigue, sleep disturbance, infections and muscular injuries could not be demonstrated in our study. Maximum sportive activity does not seem to trigger a change in riboflavin levels, therefore vitamin $B 2$ substitution with an adequate diet does not seem to make sense according to Weight et al [37]. Therapy studies are missing.

\section{Vitamin B6 (Pyridoxine)}

The prevalence of inadequate pyridoxine supply $(<5 \mu \mathrm{g} / \mathrm{l})$ in our study $(2.3 \%)$ is clearly lower compared to the results of
Manore [44] with 5 - 60\%. This may also be due to the higher pyridoxine average value $(25.13 \pm 15.24 \mu \mathrm{g} / \mathrm{l})$ of our study compared to international studies $(19.52 \pm 20.76 \mu \mathrm{g} / \mathrm{l}$ reps. $10.6 \pm 1 \mu \mathrm{g} / \mathrm{l})$ [37.44]. A national players showed higher pyridoxine values (first squad: $32.21 \pm 26.90 \mu \mathrm{g} / \mathrm{l}$ ) than all other athletes (U16/U18: $20.66 \pm 6.52 \mu \mathrm{g} / \mathrm{l}$ - adolescents $<18$ years: $23.76 \pm 18.95 \mu \mathrm{g} / \mathrm{l}$ - adults: $24.11 \pm 15.88 \mu \mathrm{g} / \mathrm{l})$. The proportion of young athletes inadequately supplied with pyridoxine $(<5 \mu \mathrm{g} / \mathrm{l})$ in our study is at $3 \%$ slightly worse than that of adults $(1.8 \%)$. There are no comparisons in the literature. In combination with magnesium, pyridoxine is responsible for the formation of important neurotransmitters (serotonin, dopamine, adrenalin, noradrenalin, melatonin) and thus for stress tolerance, coordination and concentration, as well as pain control and sleep. We could not prove that an inadequate pyridoxine supply statistically influences the factors fatigue, sleep disturbance, infections and muscular injuries. However, in $50 \%$ of the athletes, maximum sporting activity leads to a significant reduction in the pyridoxine level [44], whereby according to Weight et al [37], vitamin B6 substitution does not seem to make sense with an adequate diet. Therapy studies are also missing here.

\section{Vitamin B9 (Folic acid)}

The prevalence of inadequate folic acid supply $(<4.6$ $\mathrm{ng} / \mathrm{ml}$ ) in the present study is $10 \%$. Study comparisons are missing. In our study, the average folic acid value $(8.87 \pm 4.35$ $\mathrm{ng} / \mathrm{ml}$ ) is higher than the average value of the Weight study $(5.5 \pm 2.6 \mathrm{ng} / \mathrm{ml})$ [37]. Adolescent athletes < 18 years show higher folic acid values $(10.80 \pm 4.51 \mathrm{ng} / \mathrm{ml})$ than all other athletes (First squad: $8.79 \pm 5.92 \mathrm{ng} / \mathrm{ml}$ - U16/U18: $8.81 \pm 3.23$ $\mathrm{ng} / \mathrm{ml}$ - adults: $8.07 \pm 3.39 \mathrm{ng} / \mathrm{ml}$ ). The proportion of young athletes inadequately supplied with folic acid $(<18.7 \mathrm{ng} / \mathrm{ml})$ in our study is $8.3 \%$ lower than that of adults $(10.9 \%-\mathrm{OR}=$ $0.74-\mathrm{p}<0.068)$. Comparisons in the literature are also not available. Folic acid is responsible for cell maturation and cell differentiation of erythrocytes and thus for the prevention of megaloblastic anaemia. Statistically significant correlations between inadequate folic acid supply compared to sufficient folic acid supply with regard to the parameters fatigue, sleep disturbance, infections and muscular injuries could not be demonstrated in our study. Maximum athletic stress does not lead to a change in the folic acid level in athletes. According to Weight, et al. [37], vitamin B9 substitution with an adequate diet does not appear to be sensible. Therapy studies are also missing here.

\section{Vitamin B12 (Cobalamin)}

The prevalence of inadequate cobalamin supply $(<400$ $\mathrm{pg} / \mathrm{ml}$ ) in our study is $2.2 \%$. There are no study comparisons as well. We determined the average values $(465.72 \pm 219.57$ $\mathrm{pg} / \mathrm{ml})$ to be exactly the same as in the Weight study $(470.3 \pm$ $160.9 \mathrm{pg} / \mathrm{ml}$ ) [37]. There were no differences between the values of young athletes under the age of 18 and those of adults. The proportion of B12 inadequately supplied $(<400$ $\mathrm{pg} / \mathrm{ml}$ ) young athletes in our study is with $5.7 \%$ higher than that of adults. There is no comparison in the literature. The cobalamin as well is responsible for the cell maturation and differentiation of the erythrocytes and thus for the avoidance of megaloblastic anaemias as well as for the nerve metabolism (deep sensitivity). Statistically significant correlations between an inadequate B12 supply and a sufficient B12 supply with regard to the parameters fatigue, sleep disturbance, infections and muscular injuries could not be demonstrated in our study. Maximum athletic stress also does not lead to a change in the B12 level in athletes. According to Weight, et al. [37], vitamin B12 substitution with an adequate diet does not appear to be sensible. Therapy studies are also missing here. 


\section{Conclusion}

Professional athletes are borderline or even undersupplied on a broad basis. Adolescents $<18$ years show a considerably worse supply with micronutrients than adults. The focus is on 25-OH-VitaminD3, CoenzymQ10 and the intracellular trace elements iron, magnesium and selenium. Further studies with higher dosages and the goal of reaching the upper edge of the norm value scale must show whether a significant reduction in performance-limiting infections or muscular injuries can be achieved. The optimization of the B-vitamins targeting the upper standard margin must show whether an increase in performance by reducing lactate production under the same load is permanent and whether an improvement in sleep behaviour and a reduction in fatigue can be achieved.

\section{Acknowledgments, Conflict of Interest and Funding}

None Declared.

\section{References}

1. Rodriguez NR; DiMarco NM, Langley S (2009) American College of Sports Medicine position stand. Nutrition and athletic performance. MedSci Sports Exerc 41: 709-731.

2. Kreider RB, Wilborn CD, Taylor L, Campbell B, Almada AL, et al. (2010) ISSN exercise \& sportnutrition review: research \& recommendations. J IntSoc Sports Nutr 7: 143

3. Walsh NP, Gleeson M, Shephard RJ, Gleeson M, Woods JA, et al. (2011) Position statement. Part one: immune function and exercise. ExercImmunol Rev 17: 6-63.

4. Gleeson M, Pyne DB (2016) Respiratory inflammation and infections in high-performance athletes. Immunol CellBiol 94: 124-131.

5. Junge A, Engebretsen L, Mountjoy ML, Renström PAFH, Aubry MJ, et al. (2009) Sports injuriesduringthe Summer Olympic Games 2008. Am J Sports Med 37: 2165-2172.

6. Engebretsen L, Steffen K, Alonso JM, Aubry M, Junge A, et al. (2010) Sports injuries and illnessesduringthe Winter Olympic Games 2010. Br J Sports Med 44: 772-80.

7. Engebretsen L, Soligard T, Steffen K, Alonso JM, Aubry $M$, et al. (2013) Sports injuries and illnesses during the London Summer Olympic Games 2012. Br J Sports Med 47: 407-414.

8. Soligard T, Steffen K, Palmer-Green D, Aubry M, Grant ME, et al. (2015) Sports injuries and illnesses in theSochi 2014 Olympic Winter Games. Br J Sports Med 49: 441447.

9. Junge A, Dvorak J, Graf-Baumann T (2004) Football injuries during the World Cup 2002. Am J Sports Med 32: 23S-27S.

10. Dvorak J, Junge A, Grimm K, Kirkendall D (2007) Medical report from the 2006 FIFA World Cup Germany. Br J Sports Med41: 578-581.

11. Alonso JM, Tscholl PM, Engebretsen L, Mountjoy M, Dvorak J, et al. (2010) Occurrence of injuries and illnesses duringthe 2009 IAAF World Athletics Championships. Br J Sports Med 44: 1100-1105.

12. Alonso JM, Edouard P, Fischetto G, Adams B, Depiesse F, et al. (2012) Determination of future prevention strategies in elite track and field: analysisof Daegu 2011 IAAF championships injuries and illnesses surveillance. $\mathrm{Br} \mathrm{J}$ Sports Med 46: 505-514.

13. Alonso JM, Jacobsson $\mathrm{J}$, Timpka $\mathrm{T}$, Ronsen $\mathrm{O}$, Kajenienne A, et al. (2013) Preparticipation injury complaintis a risk factor for injury: a prospective study of the Moscow 2013 IAAF championships. Br JSports Med 49: $1118-1124$

14. Schwellnus M, Soligard T, Alonso JM, Bahr R, Clarsen $\mathrm{B}$, et al. (2016) How much is toomuch? (Part 2) International Olympic Committee consensus statement on load in sport and risk of illness. Br J Sports 50: 10431052.

15. Deakin V. Micronutrients. In: Lanham-New SA, Stear SJ, Shirreffs SM, et al. (2011) eds: Sport and Exercise Nutrition. 1st ed. Oxford, UK: Wiley-Blackwell; 2011: 66-88.

16. Hakkiday TM, Peterson NJ, Thomas JJ, Kleppinger K, Hollis BW, et al. (2011) Vitamin D status relative to diet, lifestyle, injury and illness in college athletes. MedSci Sports Exerc 43: 335-343.

17. Ruohola JP, Laaksi I, Ylikomi T, Haataja R, Mattila VM, et al. (2006) Association between serum $25(\mathrm{OH}) \mathrm{D}$ concentrations and bone stress fractures in Finnish young men. J Bone Miner Res 21: 1483-1488.

18. Kostopoulos N, Apostolidis N, Mexis D, Mikellidi A, Nomikos T (2017) Dietary intake and the markers of muscle damage in elite basket ball players after a basketball match. Journal of Physical Education and Sport 17: 394-401.

19. Gupta L, Morgan K, Gilchrist S (2017) Does Elite Sport Degrade Sleep Quality? A Systematic Review. Sports Med47: 1317-1333.

20. Ansley P, Gleeson M, Ansley L (2009) Fatigue management in the preparation of Olympic athletes. Journal of Sports Sciences 27: 1409-1420.

21. 21 Monti JM (2013) The neuro transmitters of sleep and wake, a physiological reviews series. Sleep Med Rev 17: 313-315.

22. Ogan D, Pritchett K (2013) Vitamin D and the Athlete: Risks, Recommendations and Benefits. Nutrients 5: 18561868.

23. Close GL, Russell J, Cobley JN, Owens DJ, Wilson G, et al. (2012) Assessment of vitamin D concentration in nonsupplemented professional athletes and healty adults during winter months in the UK: implications for skeletal muscle function. J Sports Sciences 31: 344-353.

24. Owens DJ, Sharples AP, Polydorou I, Alwan N, Donovan $\mathrm{T}$, et al. (2015) A systems-based investigation into vitamin $\mathrm{D}$ and skeletalmuscle repair, regeneration and hypertrophy. Am J PhysiolEndocrinolMetab 309: 10191031.

25. Quadri A, Gojanovic B, Noack P, Fuhrer C, Steuer C, et al. (2016) Seasonal variation of vitamin D levels in Swiss athletes. Swiss Sports \&Exercise Medicine 64: 19-25.

26. Sghaier-Ayadi A, Feki M, Bezrati-Ben Ayed I, Abene O, Ben Fredj MK, et al. (2015) Vitamin D status and determinants of deficiency in non-supplemented athletes during the winter months in Tunisia. Biol Sport 32: 281287.

27. Shuler FD, Wingate MK, Moore GH, Giangarra C (2012) Sports Health benefitsof Vitamin D. Sports Health 4: 496-501.

28. Fishman MP, Lombardo SJ, Kharrazi FD. Vitamin D (2016) Deficiency Among Professional Basketball Players. Orthopaedic J Sport Med 4: 1-5.

29. Varamenti E, Cherif A, Nikolovski Z, Tabben M, Jamurtas AZT, et al. (2019) Exploring possible relationships between $25(\mathrm{OH}) \mathrm{D}$ deficiency and variables related to inflammation, endothelial function, and enzymaticantioxidants in adolescent athletes: a prospectivestudy. Biol Sport 36: 113-118.

30. Kryzwanski J, Mikulski T, Krysztofiak H, Mlynczak M, Gaczynska E, et al. (2016) Seasonal Vitamin D Status in Polish Elite Athletes in Relation to Sun Exposure and Oral Supplementation. PLoS ONE 11: 1-12.

31. Backx EMP, Tieland M, Maase K, Kies AK, Mensink M, et al. (2016) The impactof 1-year vitamin D supplementation on vitamin D status in athletes: a dose-response study. European J ClinNutr 70: 1009-1014.

32. Willis KS, Smith DT, Broughton KS, Larson-Meyer DE (2012) Vitamin D status and biomarkersofinflammation in runners. J Sports Med 3: 35-42.

33. Ginde AA, Mansbach MPH, Camargo CA Jr (2009) Association Between Serum 25-Hydroxyvitamin D Level and Upper Respiratory Tract Infection in the Third 
National Health and Nutrition Examination Survey. Arch Intern Med 169: 384-390.

34. Lee $O$ (2018) Assessment ofselenium and zincstatus in female collegiate athletes. J Nutr Health 51: 121-131.

35. Fogelholm M, Laakso J, Lehto J, Ruokonen I (1991) Dietary intake and indicators of magnesium and zinc status in male athletes. Nutr Res 11: 1111-1118.

36. Molina-Lopez J, Milona JM, Chirosa LJ, Florea D, Saez L, et al. (2012) Association between erythrocyte concentrations of magnesium and zinc in high-performance handball players after dietary magnesium supplementation. Magnesium Research 25: 79-88.

37. Weight LM, Noakes TD, Labadarios D, Graves J, Jacobs P, et al. (1988) Vitamin and mineral status of trained athletes including the effects of supplementation. Am J ClinNutr 47: 186-191.

38. Finstad EW, Newhouse IJ, Lukaski HC, McAuliffe JE, Stewart CR (2001) The Effects of Magnesium Supplementation on Exercise Performance. MedSci Sports Exerc 33: 493-498.

39. Zhu YI, Haas JD (1998) Altered metabolic response of iron-depleted non anemic women during a $15-\mathrm{km}$ time trial. J ApplPhysiol 84: 1768-1775.

40. Kon M, Tanabe K, Akimoto T, Kimura F (2008) Reducing exercise-induced muscular injury in kendo athletes with supplementation of coenczyme Q10. Brit J Nutr 100: 903-909.

41. Demirci N, Beytut E (2014) Effects of oral coenzyme Q10 on preventing the accumulation of lactic acid developing during the exercise performances of endurance skiing athletes. Am J Sports Sci 2: 65-70.

42. Alf D, Schmidt ME, Siebrecht S (2013) Ubiquinol supplementation enhance speak power production in trained athletes: a double-blind, placebo controlled study. J IntSoc Sports Nutr 10: 24.

43. Choi SK, Baek SH, Choi SW (2013) The effects of endurance training and thiamine supplementation on antifatigue duringexercise. J ExercNutrBiochem 17: 189-198.

44. Manore MM (2000) Effects of physicalactivity on thiamine, riboflavin and vitamin B-6 requirements. A, J ClinNutr 72: 598s-606s.

45. Bautista-Hernandez VM, Lopez-Ascencio R, del ToroEquihua M, Vasquez C (2008) Effectof Thiamine Pyrophosphate on Levels of Serum Lactate, Maximum Oxygen Consumption and Heart Rate in Athletes Performing Aerobic Activity. J IntMed Res 36: 12201226.

46. Farrokhyar F, Tabasinejad R, Dao D, Peterson D, Ayeni OR, et al. (2015) Prevalenceof Vitamin D inadequacy in athletes: a systemic review and metaanalysis. Sports Med 45: 365-378

47. Pereira Panza VS, Diefenthaeler F, da Silva EL (2015) Benefits of dietary phytochemical supplementation on eccentric exercise-induced muscle damage: Is including antioxidants enough? Nutrition 31: 1072-1082.

48. Margaritis I, Tessier F, Prou E, Marconnet P, Marini JF (1997) Effects of endurance training on skeletalmuscle oxidative capacities with and without selenium supplementation. Trace Elem. Med. Biol 11: 37-43.

49. Heffernan SM, Horner K, De Vito G, Conway GE (2019) The Roleof Mineral and Trace Element Supplementation in Exercise and Athletic Performance: A Systematic Review. Nutrients 11: 696-729.

50. Tessier F, Margaritis I, Richard MJ, Moynot C, Marconnet P (1995) Selenium and trainingeffects on the glutathione system and aerobic performance. Med. Sci. Sports Exerc 27: 390-396.

51. Casoni I, Guglielmini C, Graziano L, Reali MG, Mazzotta D (1990) Changes of magnesium concentrations in endurance athletes. Int. J. Sports Med 11: 234-237.

52. Setaro L, Santos-Silva PR, Nakano EY, Sales $C H$, Nunes $\mathrm{N}$, et al. (2014) Magnesium status and the physical performance of volley ball players: effects of magnesium supplementation. J. Sports Sci 32: 438-444.

53. Veronese N, Berton L, Carraro S, Bolzetta F, de Rui M, et al. (2014) Effectof oral magnesium supplementation on physical performance in healthy elderly women involved in a weekly exercise program: a randomized controlledtrial. Am. J. Clin. Nutr 100: 974-981.

54. Casoni I, Borsetto A, Cavicchi A, Martinelli S, Conconi F (1985) Reduced hemoglobin concentration and red cell hemoglobinization in Italian marathon and ultramarathon runners. Int. J. Sports Med 6: 176-179.

55. DufauxB, Hoederath A, Streitberger I, Hollman W, Assman G (1981) Serum ferritin, transferrin, haptoglobin, and iron in middle-and long-distance runners, eliterowers, and professional cyclists. Int. J. Sports Med 2: 43-46.

56. Fogelholm M, Himberg JJ, Alopaeus K, Gref CG, Laakso $\mathrm{J}$, et al. (1992) Dietary and biochemical indices of nutritional status in male athletes and controls. J. Am. Coll. Nutr 11: 181-192.

57. Hemmingson $P$, Bauer M, Birgeghd G (1991) Iron status in elite skiers. Scand. J. Med. Sci. Sports 1: 174-179.

58. Magnusson B, Hallberg L, Rossander L, Swolin B (1984) Iron metabolism and "sports anemia". II. A hematological comparison of elite runners and control subjects. Acta Med. Scand 216: 157-164.

59. Pate RR, Miller BJ, Davis JM, Slentz CA, Klingshim LA (1993) Iron status of female runners. Int. J. Sport Nutr 3: 222-231.

60. Risser WL, Lee EJ, Poindexter HB, West MS, Pivarnik JM, et al. (1988) Hickson. Iron deficiency in femaleathletes: Itsprevalence and impact on performance. Med. Sci. Sports Exerc. 1988: 20, 116-121.

61. Erpenbach K, Mücke S (2017) Mitochondriale Ernährung. Sportärztezeitung 4: 88-91. 